\title{
Dynamics of Branched Polymers: A Combined Study by Molecular Dynamics Simulations and Tube Theory
}

\author{
L.G.D Hawke, ${ }^{1}$ P. Bačová, ${ }^{2}$ D.J. Read,${ }^{1}$ and A.J. Moreno ${ }^{3,2}$ \\ ${ }^{1}$ Department of Applied Mathematics, University of Leeds, LS2 9JT Leeds, UK \\ ${ }^{2}$ Donostia International Physics Center, Apartado 1072, 20080 San Sebastián, Spain \\ ${ }^{3}$ Centro de Física de Materiales (CSIC, UPV/EHU) and Materials Physics Center MPC, \\ Paseo Manuel de Lardizábal 5, E-20018 San Sebastián, Spain
}

\begin{abstract}
We present large-scale computer simulations of entangled polymers with symmetric star-like and Cayley tree-like architectures. Unlike the usual observation for repational behaviour of linear chains, the simulated systems exhibit a strong dispersion, over several decades, of the relaxation times after the local reptative ('Rouse in tube') regime. Relaxation is dramatically slowed down by approaching the branch point from the outer segments. This is consistent with the expected retraction mechanism for strongly entangled branched polymers. In order to describe fluctuations around the branch point, we introduce a Rouse-like model adapted to star-like polymers and incorporate entanglements by means of localizing springs. Model predictions for localization of the branch point are compared with simulations with fixed arm ends, which suppress retraction and tube dilution. Strikingly, the simulations reveal a localization of the branch point weaker than expected. This suggests the presence of early constraint-release effects that are not captured by the standard mechanism of tube dilution. We quantify, as a function of time, the strength of such effects and the fraction of relaxed material directly from the simulations with free ends. This allows us to renormalize the tube diameter and entanglement time in our model as time-dependent quantities. With this renormalization, the model provides an excellent description of the early relaxation of the branch point.
\end{abstract}

PACS numbers:

\section{INTRODUCTION}

\section{SIMULATION DETAILS}

The simulated systems were modelled according to the well-known scheme proposed by Grest and Kremer [1]. In this coarse-grained model, the chains are represented as strings of beads ('monomers') connected by springs, with generic interactions describing excluded volume and connectivity (see below). Fig. 1 shows a schematic representation of the architectures of the simulated systems. These are linear chains, 3-arm symmetric stars and Cayley trees. The numbers associated to each backbone and branch in Fig. 1 represent the respective number, $Z$, of entanglement segments. The corresponding number of monomers is $Z N_{\mathrm{e}}$, with $N_{\mathrm{e}}$ the entanglement length, i.e., the number of monomers per entanglement segment. For the specific model simulated in this work, we have $N_{\mathrm{e}}=25$, though it should be noted that there is some uncertainty in the value depending on how it is estimated (see below).

As can be seen in Fig. 1 the maximum distance between two outermost monomers of different branches in the stars and Cayley trees is, in all cases, $Z=16$ entanglement segments (400 monomers). This is identical to the length of the linear chains. Simulation of these three selected systems can provide useful information about the effect of branching on the chain dynamics. Indeed, the 3 -arm star is obtained by attaching a branch of length $Z=8$ to the center of the linear chain. Likewise, the Cayley tree is obtained by attaching branches of length
$Z=2$ to the centers of the star arms.

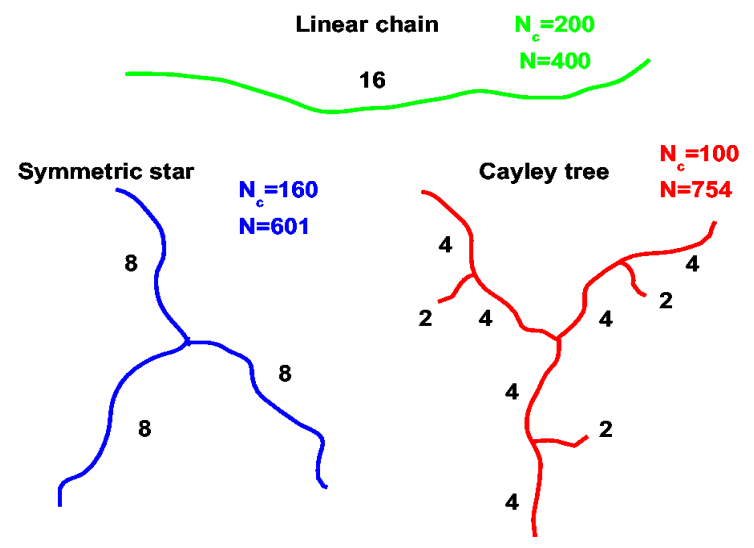

FIG. 1: Illustration of the simulated systems: $N_{c}$ denotes the number of the polymers in the simulation box. $N$ is the number of beads per macromolecule. The numbers labelling backbones and branches denote the respective lengths, expressed as multiples of the entanglement segment $N_{\mathrm{e}}=25$.

As aforementioned, in the Grest-Kremer model the monomeric units are represented by beads. The excluded volume interaction is given by a purely repulsive Lennard-Jones (LJ) potential:

$$
U_{\mathrm{LJ}}(r)= \begin{cases}4 \epsilon\left[\left(\frac{\sigma}{r}\right)^{12}-\left(\frac{\sigma}{r}\right)^{6}+\frac{1}{4}\right] & \text { for } r \leq r_{\mathrm{c}} \\ 0 & \text { for } r>r_{\mathrm{c}}\end{cases}
$$


with a cut-off distance $r_{\mathrm{c}}=2^{1 / 6} \sigma$. The LJ diameter $\sigma$ is the length unit of the model. The temperatute unit is $\epsilon / k_{\mathrm{B}}$, with $k_{\mathrm{B}}$ the Boltzmann constant. The time unit is given by $\tau_{0}=\left(m_{0} \sigma^{2} / \epsilon\right)^{1 / 2}$, with $m_{0}$ the monomer mass. Connectivity between consecutive beads is provided by a finite-extension nonlinear elastic (FENE) potential:

$$
U_{\mathrm{F}}=-\frac{1}{2} K_{\mathrm{F}} R_{\mathrm{F}}^{2} \ln \left[1-\left(\frac{r}{R_{\mathrm{F}}}\right)^{2}\right]
$$

We use $K_{\mathrm{F}}=30 \epsilon / \sigma^{2}$ for the spring constant and $R_{\mathrm{F}}=$ $1.5 \sigma$ for the maximum bond length. Furthermore, a certain degree of chain stiffness is introduced by applying a bending potential:

$$
U_{\text {bend }}(\theta)=k_{\theta}(1-\cos \theta) \text {, }
$$

where $\theta$ is the bending angle between three consecutive monomers $(\theta=0$ for a rod). We have used a bending constant $k_{\theta}=2 \epsilon$. With this choice, long linear chains have a characteristic ratio $C_{\infty}=\lim _{N \rightarrow \infty}\left\langle R_{\mathrm{e}}^{2}\right\rangle / N=3.4$ at the investigated temperature and density (see below). In the former expression $R_{\mathrm{e}}$ and $N$ are the end-to-end distance and the number of monomers per chain, respectively. The value $C_{\infty}=3.4$ is higher than in flexible bead-spring chains $\left(k_{\theta}=0, C_{\infty}=1.76\right)$ and lower than in common polymers as polyisoprene or polybutadiene $\left(C_{\infty} \sim 5\right)$.

The introduction of some local stiffness has the advantage of decreasing the entanglement length $N_{\mathrm{e}}$. This reduces computational cost since we can simulate, with the same chain length $N$, more strongly entangled systems than by using flexible chains. For the semiflexible chains investigated in this work $\left(k_{\theta}=2 \epsilon\right)$ one finds $N_{e} \approx 25$. A primitive path analysis gives $N_{e}^{P P}=23$ [2], whereas an estimation from the monomer mean square displacement (MSD) gives $N_{e}^{M S D}=27$ [3]. These are considerably smaller than the values found for flexible chains $\left(k_{\theta}=0\right.$; $\left.N_{e}^{P P}=65, N_{e}^{M S D}=50\right)[4]$.

On the other hand, we aim to compare simulation results with tube-based models that are formulated for Gaussian chains. Therefore the chain stiffness introduced by the bending potential should not be too strong, since local deviations from Gaussianity should not persist at contour distances beyond the entanglement length. The simulated case constitutes a good compromise between a relatively short entanglement length and a short range of non-Gaussian effects. Indeed we have checked that, if $R(|n-m|)$ is the distance between the $n$th and $m$ th monomer, the limit $\left\langle R^{2}(|n-m|)\right\rangle /|n-m| \approx C_{\infty}$ is already found for $|n-m| \sim N_{e}$.

All the simulations were performed at temperature $T=\epsilon / k_{\mathrm{B}}$ and number density $\rho=\left(N N_{c}\right) / V=0.85 \sigma^{-3}$, with $N_{c}$ the number of macromolecules in the simulation box of volume $V$, and $N$ the number of monomers per macromolecule. The former density corresponds to melt conditions in bead-spring polymers. We used boxes with a total of 80000,96160 and 75400 monomers for the systems of linear chains, stars and Cayley trees respectively. Before the production run, the system has to be equilibrated properly. The time scales for equilibration of the long and branched macromolecules simulated here are far beyond the current computational capabilities by using standard MD. However, efficient methods combining Monte Carlo (MC) and MD simulations have been developed to equilibrate large-scale conformations with low computational cost. We used a very similar method to that proposed by Auhl et. al. [5]. Thus we first equilibrated, by standard MD, systems of small linear chains and 3-arm symmetric stars, with a length of one entanglement per linear backbone or star arm. These were used as building blocks to construct the long branched macromolecules with the desired architecture. The bending angles at the junction points of different building blocks were selected so that the macromolecular conformation obeyed the correct target function [5] $C\left([n-m \mid)=\left\langle R^{2}(|n-m|)\right\rangle /|n-m|\right.$ (see Ref. [5] for details).

Afterwards, we placed randomly the generated macromolecules in the simulation box, and followed the prepacking procedure proposed in [5]. This consists of an MC simulation in which the macromolecules are treated as rigid objects performing large-scale motions (rotations, translations, reflections...) that are accepted only when they reduce the local density fluctuations [5]. After a significant reduction of the inhomogeneities, we performed a short MD run $\left(\sim 10^{6}\right.$ steps, with a time step $\left.\Delta t=0.001 \tau_{0}\right)$ in which force-capping [5] was applied to the LJ interaction of eq. 1 . The capping radius was slowly reduced and finally the full LJ interaction was switched on. The equilibration was finished with a standard MD run of about $10^{8}$ time steps. We checked that the obtained configurations obeyed the target function $C([n-m \mid)$. The time step for this last equilibration run as well as for the production runs was $\Delta t=0.01 \tau_{0}$.

The MC simulations were perfomed by using a homemade code. The MD simulations were performed by using the ESPResSo simulation package [6]. All the runs were perfomed at constant volume. In the MD runs, the temperature was controlled by the Langevin thermostat with a friction constant $\Gamma=0.5 m_{0} / \tau_{0}$ and the equations of motion were integrated by using the velocity-Verlet algorithm. The equilibration runs were performed serially (for MC) or with low paralellization (for MD). The production MD runs extended over unusually long time scales (up to $3 \times 10^{9}$ time steps) and required high parallelization. The estimated total CPU time for the production runs was of $10^{6}$ hours.

\section{INITIAL OBSERVATIONS FROM SIMULATION RESULTS}

The time evolution of the monomer mean square displacement (MSD), $\left\langle\Delta r^{2}(t)\right\rangle$, provides valuable information about the microscopic dynamics of the system. This 
quantity, which is often difficult to be accessed in experiments, can be easily computed from the simulation data. Moreover, by computing the MSD of specific segments along the macromolecule, we may shed light on the role of the macromolecular architecture on the internal relaxation mechanisms. In our analysis of the simulation data, we have divided the macromolecules into segments of length equal to one entanglement $\left(N_{\mathrm{e}}=25\right.$ monomers). The corresponding MSD of different segments in the three investigated systems are shown in Figs. 2 and 3. The notations in the legends for the different data sets must be understood as follows. We treat the linear chain as a 2 -arm star with the branch point in the center of the backbone, i.e., the arms have $Z=8$ entanglement segments, as in the 3 -arm stars. We label the entanglement segments in each arm of the linear chains and stars as $e=1,2, \ldots 8$, by following the path from the branch point to the outermost monomer in the same arm. Obviously, for each entanglement segment in a given arm there are, by symmetry, other equivalent segments in the other arms and accordingly the corresponding MSD is averaged over them. In the case of the Cayley trees we do not include in Fig. 3 the data for the short side branches $(Z=2)$, and the entanglement segments are labelled in the same way as in the linear chains and stars. Thus, we label the segments as $e=1,2, \ldots 8$, by following the path of length $Z=4$ from the central branch point to one of the three outer ones, and from there to the outermost monomer in the same branch of $Z=4$ (see Fig. 1). Again, the MSD is averaged over equivalent segments in the three long arms.

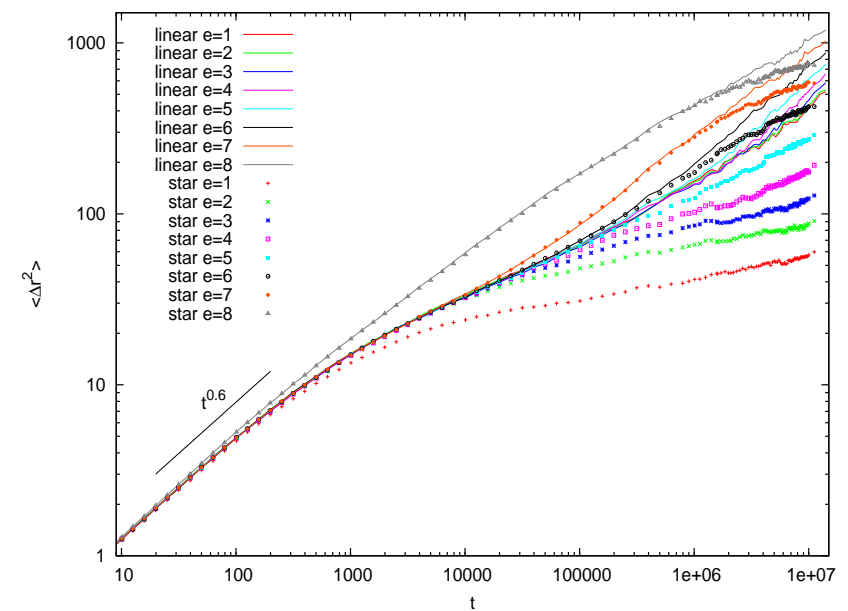

FIG. 2: MSD of the entanglement segments (see text) in the linear chains and symmetric stars

In Fig. 2 we compare data of the linear chains and symmetric stars. In Fig. 3 the comparison is done for the linear chains and Cayley trees. Up to the entanglement time $\tau_{\mathrm{e}} \approx 1800$ (see Ref. [3]), the MSD of the different segments follow Rouse behaviour. The data are better described by an effective power-law $\left\langle\Delta r^{2}\right\rangle \sim t^{0.6}$ than by the strictly Rouse-like behaviour $\left\langle\Delta r^{2}\right\rangle \sim t^{1 / 2}$. This

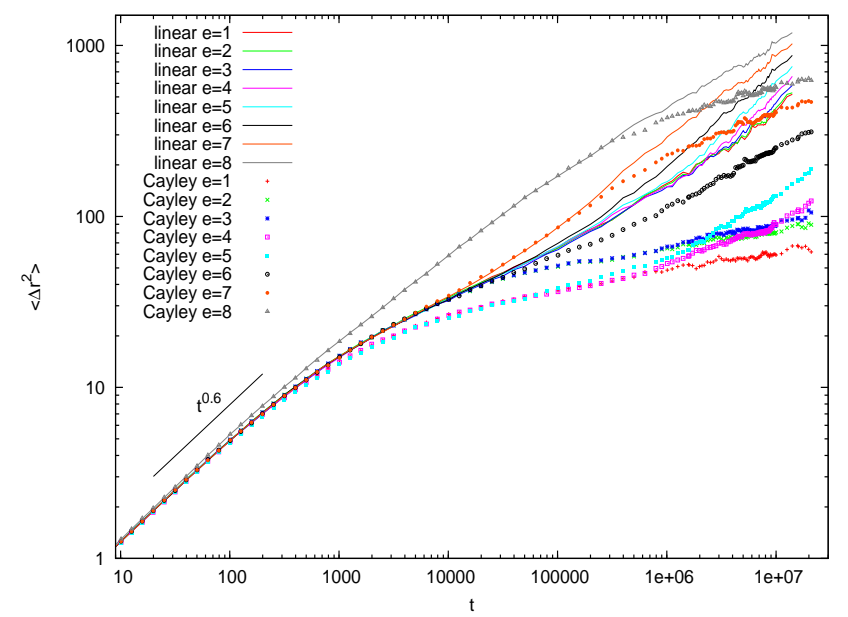

FIG. 3: MSD of the entanglement segments (see text) in the linear chains and Cayley trees

small difference may originate from non-Gaussian correlations (not included in the Rouse model) at $N<N_{\mathrm{e}}$, which are related to the semiflexible character introduced by the bending potential (see above).

At the entanglement time $\tau_{\mathrm{e}} \approx 1800$ the different segments start to probe the topological constraints, and the MSD progressively deviates from the Rouse behaviour. In the usual picture for linear chains, the initial fluctuations of the monomer along the primitive path are described as Rouse dynamics of the curvilinear coordinate ('Rouse in tube' dynamics). The consequence of this for the real-space monomer dynamics is that the MSD scales as $\left\langle\Delta r^{2}(t)\right\rangle \sim t^{1 / 4}$. As aforementioned, the Rouse regime $\left\langle\Delta r^{2}(t)\right\rangle \sim t^{x}$ in our system is characterized by an exponent $x=0.6$ instead of the ideal value $x=1 / 2$. Accordingly, we may expect that the characteristic exponent for the 'Rouse in tube' dynamics is $x=0.3$ instead of the ideal value $x=1 / 4$. Figs. 4 and 5 show the ratio $\left\langle\Delta r^{2}(t)\right\rangle / t^{0.3}$. In this representation the 'Rouse in tube' regime is recognized as a plateau for $t>\tau_{\mathrm{e}}$. Most of the segments in the three investigated architectures exhibit this behaviour for at least a portion of the time window $\tau_{\mathrm{e}}<t<\tau_{\mathrm{R}}$, where $\tau_{\mathrm{R}}$ is the Rouse time, i.e., the time scale for the longest internal chain modes [7]. This can be estimated as $\tau_{\mathrm{R}}=\tau_{\mathrm{e}}\left(N_{a} / N_{e}\right)^{2} \approx 10^{5}$, with $N_{a}=200$ the number of monomers per long arm. Data of some specific segments do not obey the mentioned overlap, namely the outermost segments $(e=8)$ and the segments directly attached to the branch points $(e=1$ in stars and Cayley trees, as well as $e=4$ and 5 in Cayley trees).

In the case of the outermost segments $e=8$, the data reveal a much faster behaviour than the plateau regime $\left\langle\Delta r^{2}(t)\right\rangle / t^{0.3} \sim t^{0}$. This can be understood as follows. The intramolecular conformation can perform strong fluctuations in the neighborhood of the free ends, since the segments there are weakly affected by the topological constraints. As a consequence, the primitive path near the chain ends is almost fully relaxed by simple 


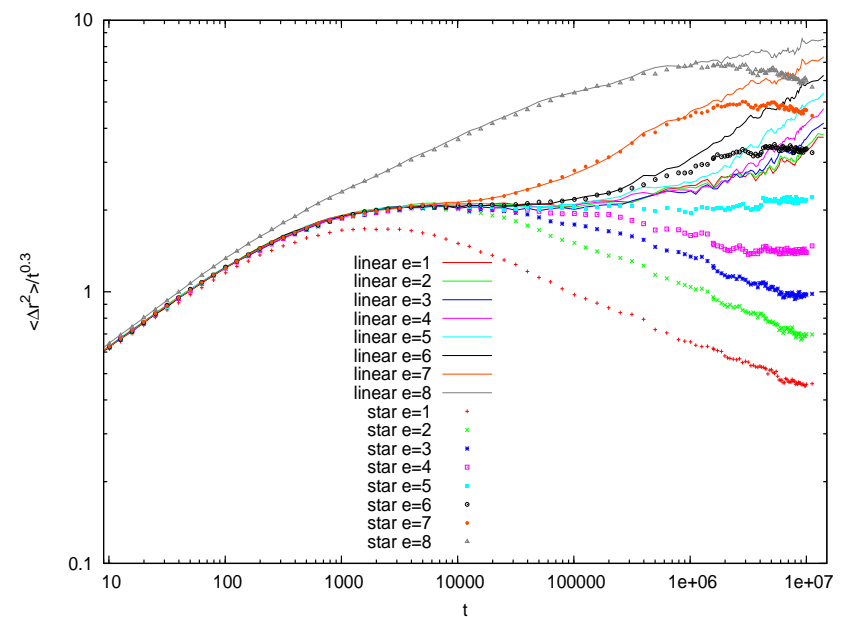

FIG. 4: MSD of the entanglement segments in the linear chains and symmetric stars normalized by $t^{0.3}$

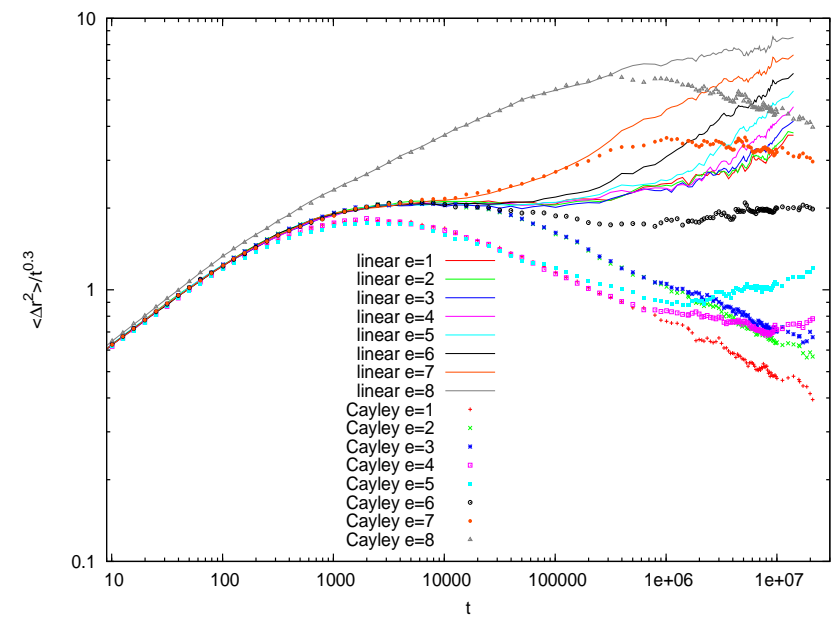

FIG. 5: MSD of the entanglement segments in the linear chains and Cayley trees normalized by $t^{0.3}$

Rouse dynamics at $t<\tau_{\mathrm{e}}$. As can be seen in Figs. 2 and 3 the initial Rouse behaviour is indeed weakly perturbed up to time scales of $t \sim \tau_{\mathrm{R}} \gg \tau_{\mathrm{e}}$. Moreover this feature does not depend on the specific intramolecular arquitecture up to long time scales. Thus, the MSD of the outermost segments $e=7,8$ of the linear chains is indistinguishable from the corresponding data for the stars and Cayley trees up to times of $t \geq \tau_{\mathrm{R}}$. In summary, for times $t<\tau_{\mathrm{R}}$, the outermost segments do not probe the specific relaxation mechanisms associated to each intramolecular architecture. For longer times, the MSD of the outer segments of both the star and Cayley tree architectures is smaller than that of the corresponding segments of the linear chain. This is because the outer segments remain attached to more slowly relaxing inner sections of chain; although they can easily escape their own tube constraints, they cannot move large distances because of entanglement constraints on the rest of the chain.

In the case of the segments directly attached to the branch points, the data exhibit a clear slowing down with respect to the 'Rouse in tube' dynamics of other segments. We will see that this effect esentially originates from the threefold connectivity of the branch point, and that can still be explained by considering local Rouse motion in a tube (see Section IV B).

Thus, with the mentioned exception of the outermost segments, the MSD exhibits universal 'Rouse in tube' dynamics over a certain time window after the entanglement time, even if the segments are not placed in linear chains but in arms of branched architectures. However, rather evident differences between the different architectures emerge at longer times $t>\tau_{\mathrm{R}}$. Thus, the overlap in the MSD of the inner segments $(e<3)$ persists in the linear chains, and the scaling behaviour changes from $\left\langle\Delta r^{2}(t)\right\rangle \sim t^{0.3}$ to $\left\langle\Delta r^{2}(t)\right\rangle \sim t^{1 / 2}$. These features are consistent with the expected reptational mechanism for inner segments at long times. In contrast with the observation for linear chains, the MSD for $t>\tau_{\mathrm{R}}$ spreads out dramatically in the stars. Since the three long arms $(Z=8)$ are equivalent and relax in the same time scale, there is not a common tube over which the whole star can reptate at long times. Instead, relaxation occurs by deep contour length fluctuations (arm retraction). Because this mechanism involves a large entropic cost, the mobility of the segments in the stars is progressively reduced as the branch point is approached. The data in Fig. 2 evidence a broad distribution of relaxation times along the arm contour. Thus, at the end of the simulation $\left(t \sim 2 \times 10^{7}\right)$ the difference between the MSD of the outermost and innermost segments of the star arms is about a factor 10 . The expected ultimate merging of all data sets will occur at time scales far beyond the simulation limits.

For the same reason discussed above, reptation in Cayley tree is not possible either. Again, relaxation occurs via arm retraction, leading to variation in mobility along the arm contour. However, unlike in stars, this variation is not monotonic. Thus, in a broad dynamic window the segments directly attached to the outer branch points $(e=4,5)$ are more restricted than some inner segments that are closer to the central branch point $(e=2,3)$. This behaviour is found at time scales before full relaxation of the short side branches. This can be estimated from the normalized orientational correlator (not shown) $P(t)=\left\langle\mathbf{e}_{\mathbf{s}}(t) \cdot \mathbf{e}_{\mathbf{s}}(0)\right\rangle /\left\langle e_{s}^{2}(0)\right\rangle$, where $\mathbf{e}_{\mathbf{s}}$ is the end-to-end vector of the short side branch. We find $P(t)<0.05$ for $t>10^{6}$. At much longer times after full relaxation of the short branches, the MSD of the different segments in the Cayley trees recovers the monotonic behaviour observed in the stars (see Figs. 2 and 4). This feature is consistent with the idea of hierarchical relaxation. After relaxation of the short side branches, they act as source of extra friction for motion of the main arms, and the Cayley tree is reduced to an effective symmetric star. 


\section{THEORY}

\section{A. Rouse dynamics}

To provide a basic model with which to compare simulation results, we have derived expressions for the MSD of monomers in the Rouse model for star polymer architectures. These expressions have been obtained for both free chains and, in order to model localisation due to entanglements, chains where monomers are localised by a quadratic potential [8-13] (see next subsection). A similar calculation was attempted for linear chains by Vilgis and Boué [10] but their expressions do not reduce to the Gaussian chain result at equilibrium because they do not include the contribution of the mean path. Our equations derived below correct this point, and can be used for linear chains if these are treated as two-arm stars. By using the continuous chain Rouse model, we are explicitly ignoring some complications inherent to the molecular dynamics model, such as the discrete nature of the beads or the bending potential (eq 3). Nevertheless, the expressions derived here provide a starting point for the analysis of monomer motion near branch points in the MD simulations. It must be stressed that these expressions refer only to local branch point motion within the tube and not to the diffusive steps (curvilinear hopping) that a branch point undertakes after an arm has fully escaped from its tube.

For an unentangled star polymer, the Langevin equation and the free energy read, respectively [7, 14]

$$
\begin{gathered}
\zeta_{0} \frac{\partial \mathbf{r}_{\alpha, \ell, t}}{\partial t}=k \frac{\partial^{2} \mathbf{r}_{\alpha, \ell, t}}{\partial \ell^{2}}+\mathbf{g}(\alpha, \ell, t) \\
F_{R}=\frac{k}{2} \sum_{\alpha=1}^{f} \sum_{\ell=0}^{N_{a}}\left(\mathbf{r}_{\alpha, \ell+1, t}-\mathbf{r}_{\alpha, \ell, t}\right)^{2}= \\
\frac{k}{2} \sum_{\alpha=1}^{f} \int_{0}^{N_{a}}\left(\frac{\partial \mathbf{r}_{\alpha, \ell, t}}{\partial \ell}\right)^{2} d \ell
\end{gathered}
$$

where $\mathbf{r}=\mathbf{r}_{\alpha, \ell, t}$ is the position vector of the $\ell$ th segment in the $\operatorname{arm} \alpha$ at time $t$. The Rouse segments in each arm are labelled $\ell=0,1, . . N_{a}$ starting from the branch point where $\ell=0$ and ending at the arm tip where $\ell=N_{a}$ (Fig. 6- left).

The drag is uniformly distributed all over the chain with each segment carrying an effective drag of $\zeta_{0}$. The factor $k=3 k_{B} T b^{-2}$ is the entropic spring constant, where $b$ is the segmental length. The term $\mathbf{g}(\alpha, \ell, t)$ is the Brownian force on the $\ell$ th segment of the arm $\alpha$ with averages $\langle\mathbf{g}(\alpha, \ell, t)\rangle=0$ and $\left\langle g_{\mu}(\alpha, \ell, t) g_{\nu}\left(\beta, \ell^{\prime}, t^{\prime}\right)\right\rangle=$ $2 \zeta_{0} k_{B} T \delta\left(\ell-\ell^{\prime}\right) \delta\left(t-t^{\prime}\right) \delta_{\alpha \beta} \delta_{\mu \nu}$. Indices $\mu$ and $\nu$ denote cartesian coordinates while $\alpha$ and $\beta$ are used to label different arms. The boundary conditions of eq $4 \mathrm{a}$ are determined by the specific polymer architecture (linear, star, Cayley, comb, etc.).

In Appendix A we present the appropriate boundary conditions for a symmetric star with $f$ arms, and calculate the MSD of two segments placed at the same or

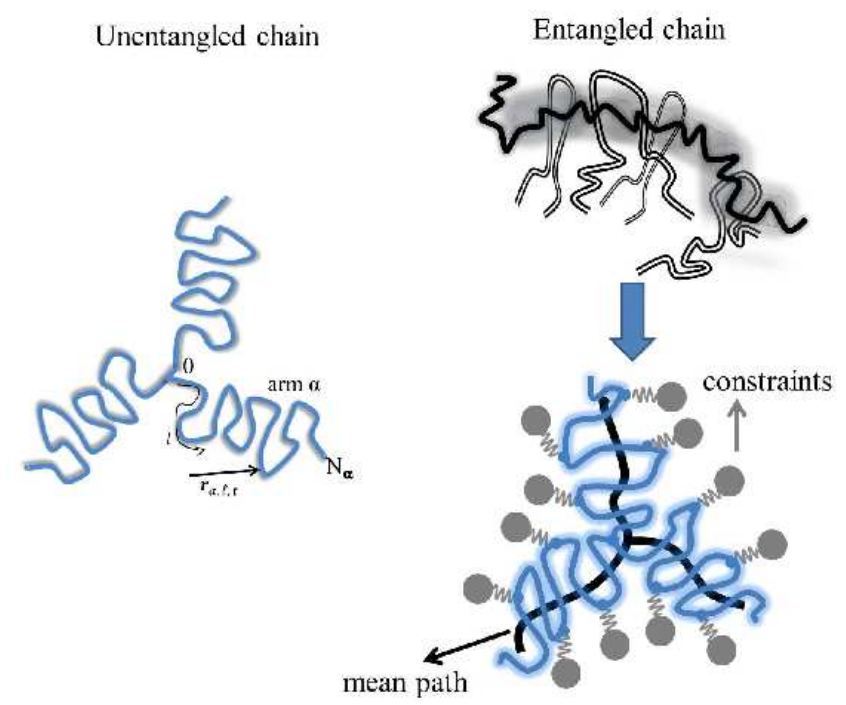

FIG. 6: Left: Schematic illustration of an unentangled star. The position vector $\mathbf{r}=\mathbf{r}_{\alpha, \ell, t}$ of the $\ell t h$ segment in arm $\alpha$ at time $t$ is shown. Right: The entanglements are modeled by localising springs (constraints). The thick black line shows the mean path.

different arms. We have made the approximation that the fast Rouse modes (small wavelengths) dominate the dynamics. Therefore, the expressions are strictly valid for $t \ll \tau_{R_{a}}$ where $\tau_{R_{a}}$ is the Rouse relaxation time of an arm given by $\tau_{R_{a}}=\zeta_{0} b^{2} N_{a}^{2}\left(3 \pi^{2} k_{B} T\right)^{-1}$. The obtained results are presented in Table I. In these expressions, $\Phi(x)$ is the error function given by $\Phi(x)=\frac{2}{\sqrt{\pi}} \int_{0}^{x} e^{-u^{2}} d u$ and $\widetilde{t}_{R_{a}}=\left|t-t^{\prime}\right| \tau_{R_{a}}^{-1}$ is the time normalized by $\tau_{R_{a}}$. The terms $\left\langle\left(\mathbf{r}_{\alpha, \ell, t}-\mathbf{r}_{\alpha, \ell^{\prime}, t^{\prime}}\right)^{2}\right\rangle$ and $\left\langle\left(\mathbf{r}_{\alpha, \ell, t}-\mathbf{r}_{\beta, \ell^{\prime}, t^{\prime}}\right)^{2}\right\rangle$ refer to the MSD of segments in the same and in different arms respectively. The expression for the segmental self-motion $\left(\alpha=\beta, \ell=\ell^{\prime}\right)$ is given in the third row of table I. This expression reduces to $\left\langle\left(\mathbf{r}_{\alpha, 0, t}-\mathbf{r}_{\alpha, 0, t^{\prime}}\right)^{2}\right\rangle=\frac{2}{f} \frac{2 N_{a} b^{2}}{\pi^{1.5}} \sqrt{\widetilde{t}_{R_{a}}}$ (fourth row of table I) for the branch point, which is lower by a factor of $2 / f$ compared to the segmental motion in a linear chain of polymerization degree $N_{a}$ [7, 14]. The expressions of Table I are consistent in the limit case of linear chains. Indeed if we set $f=2$ they provide the well-known Rouse behaviour for the segmental motion of unentangled linear chains. Moreover, at equilibrium $\left(t=t^{\prime}\right)$ the Gaussian chain limit is recovered independently of $f$. Indeed the terms $\left\langle\left(\mathbf{r}_{\alpha, \ell, t}-\mathbf{r}_{\alpha, \ell^{\prime}, t^{\prime}}\right)^{2}\right\rangle$ and $\left\langle\left(\mathbf{r}_{\alpha, \ell, t}-\mathbf{r}_{\beta, \ell^{\prime}, t^{\prime}}\right)^{2}\right\rangle$ reduce, for $t=t^{\prime}$, to the Gaussian chain result, $b^{2}\left|\ell-\ell^{\prime}\right|$ and $b^{2}\left(\ell+\ell^{\prime}\right)$ respectively.

\section{B. Entangled dynamics}

In a polymer melt, the entanglements imposed by the surrounding chains on a test chain localise it in space. This effect is not incorporated in eq $4 \mathrm{a}$. Therefore, for 
TABLE I: MSD of unentangled stars

\begin{tabular}{|c|c|}
\hline MSD & Expression \\
\hline$\left\langle\left(\mathbf{r}_{\alpha, \ell, t}-\mathbf{r}_{\alpha, \ell^{\prime}, t^{\prime}}\right)^{2}\right\rangle$ & 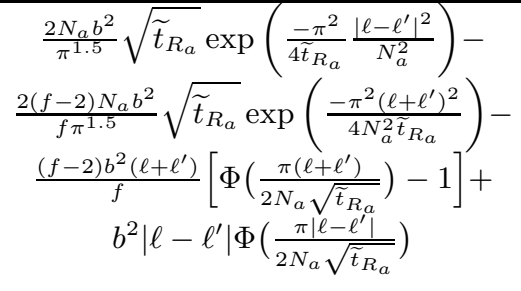 \\
\hline$\left\langle\left(\mathbf{r}_{\alpha, \ell, t}-\mathbf{r}_{\beta, \ell^{\prime}, t^{\prime}}\right)^{2}\right\rangle$ & $\begin{array}{l}\frac{b^{2}\left(\ell+\ell^{\prime}\right)}{f}\left[(f-2)+2 \Phi\left(\frac{\pi\left(\ell+\ell^{\prime}\right)}{2 N_{a} \sqrt{\widetilde{t}_{R_{a}}}}\right)\right]+ \\
\frac{4 N_{a} b^{2}}{f \pi^{1.5}} \sqrt{\widetilde{t}_{R_{a}}} \exp \left(\frac{-\pi^{2}\left(\ell+\ell^{\prime}\right)^{2}}{4 N_{a}^{2} \widetilde{t}_{R_{a}}}\right)\end{array}$ \\
\hline$\left\langle\left(\mathbf{r}_{\alpha, \ell, t}-\mathbf{r}_{\alpha, \ell, t^{\prime}}\right)^{2}\right\rangle$ & $\begin{array}{c}2 b^{2} \ell\left(\frac{f-2}{f}\right)\left[1-\Phi\left(\frac{\pi \ell}{\sqrt{\widetilde{t}_{R_{a}}} N_{a}}\right)\right]+ \\
\frac{2 N_{a} b^{2}}{\pi^{1.5}} \sqrt{\widetilde{t}_{R_{a}}}\left[1-\left(\frac{f-2}{f}\right) \exp \left(\frac{-\pi^{2} \ell^{2}}{t_{R_{a}} N_{a}^{2}}\right)\right]\end{array}$ \\
\hline$\left\langle\left(\mathbf{r}_{\alpha, 0, t}-\mathbf{r}_{\alpha, 0, t^{\prime}}\right)^{2}\right\rangle$ & $\frac{2}{f} \frac{2 N_{a} b^{2}}{\pi^{1.5}} \sqrt{\widetilde{t}_{R_{a}}}$ \\
\hline
\end{tabular}

timescales bigger than the entanglement time $\tau_{e}$ an alternative model is required. Following on from the earlier works of [8-12] and Read et al. [13], in order to model the entanglement effect, we localise each monomer $(\alpha, \ell)$ of a Rouse chain by a harmonic potential centered at a fixed point $\mathbf{R}_{\alpha, \ell}$ (Fig. 6 right). The strength of the potential is parameterised by $h_{s}$. One may consider the potential as a virtual anchoring chain with $N_{s}$ monomers, where $N_{s}=h_{s}^{-1}$. The Langevin equation and the free energy in this model read, respectively:

$\zeta_{0} \frac{\partial \mathbf{r}_{\alpha, \ell, t}}{\partial t}=k \frac{\partial^{2} \mathbf{r}_{\alpha, \ell, t}}{\partial \ell^{2}}+k h_{s}\left(\mathbf{R}_{\alpha, l}-\mathbf{r}_{\alpha, \ell, t}\right)+\mathbf{g}(a, \ell, t)$

$F=\frac{k}{2} \sum_{\alpha=1}^{f} \sum_{\ell=0}^{N_{a}}\left[\left(\mathbf{r}_{\alpha, \ell+1, t}-\mathbf{r}_{\alpha, \ell, t}\right)^{2}+h_{s}\left(\mathbf{R}_{\alpha, l}-\mathbf{r}_{\alpha, \ell, t}\right)^{2}\right]$

where the additional terms involving $h_{s}$ arise from the localising potential. Each segment fluctuates about a position averaged over the entanglement relaxation time $\tau_{e}$ (since the $\mathbf{R}_{\alpha, \ell}$ 's are fixed). Therefore, the position vector of each segment can be expressed as

$$
\mathbf{r}_{\alpha, \ell, t}=\widehat{\mathbf{r}}_{\alpha, \ell}+\boldsymbol{\Delta}_{\alpha, \ell, t},
$$

where $\widehat{\mathbf{r}}_{\alpha, \ell}$ is the time-independent average position of the $\ell$ th Rouse segment in the arm $\alpha$ and $\boldsymbol{\Delta}_{\alpha, \ell, t}$ denotes the fluctuations about the average position. When all average positions are connected the mean path is obtained. As shown in Ref. [13], the mean path is obtained from eq 5b by requiring that $\partial F / \partial \mathbf{r}=0$ at $\mathbf{r}=\widehat{\mathbf{r}}_{\alpha, \ell}$, which yields

$$
\begin{aligned}
\mathbf{R}_{\alpha, \ell} & =\widehat{\mathbf{r}}_{\alpha, \ell}-\frac{1}{h_{s}}\left(\widehat{\mathbf{r}}_{\alpha, \ell+1}+\widehat{\mathbf{r}}_{\alpha, \ell-1}-2 \widehat{\mathbf{r}}_{\alpha, \ell}\right) \\
& =\widehat{\mathbf{r}}_{\alpha, \ell}-\frac{1}{h_{s}} \frac{\partial^{2} \widehat{\mathbf{r}}_{\alpha, \ell}}{\partial \ell^{2}}
\end{aligned}
$$

When eq 7 is substituted into eq $5 \mathrm{~b}$ the free energy can be rewritten, in the continuous chain limit, as a sum of two independent contributions:

$$
\begin{aligned}
& F=\underbrace{\frac{k}{2} \sum_{\alpha=1}^{f} \int_{0}^{N_{a}}\left[\left(\frac{\partial \widehat{\mathbf{r}}_{\alpha, \ell}}{\partial \ell}\right)^{2}+\frac{1}{h_{s}}\left(\frac{\partial^{2} \widehat{\mathbf{r}}_{\alpha, \ell}}{\partial \ell^{2}}\right)^{2}\right] d \ell}_{\text {mean path }}+ \\
& \underbrace{\frac{k}{2} \sum_{\alpha=1}^{f} \int_{0}^{N_{a}}\left[\left(\frac{\partial \boldsymbol{\Delta}_{\alpha, \ell, t}}{\partial \ell}\right)^{2}+h_{s} \boldsymbol{\Delta}_{\alpha, \ell, t}^{2}\right] d \ell}_{\text {fluctuations }}
\end{aligned}
$$

one depending only on the mean path (first term) and another depending only on the fluctuations about the mean path (second term). From the above equation it is apparent that the mean path contribution contains the usual Gaussian chain stretching energy term, $(k / 2)\left(\partial \widehat{\mathbf{r}}_{\alpha, \ell} / \partial \ell\right)^{2}$, and a second term $(k / 2) h_{s}^{-1}\left(\partial^{2} \widehat{\mathbf{r}}_{\alpha, \ell} / \partial \ell^{2}\right)^{2}$, which penalises bending of the mean path. Eq 8 itself is adequate enough for the description of the equilibrium configuration of the chain, but eq 8 does not provide any information on the conformational changes of the chain as a function of time.

We need to examine the time evolution of the fluctuation term $\boldsymbol{\Delta}_{a, \ell, t}$. Substitution of eq 7 in eq 5 a gives the appropriate Langevin equation

$$
\zeta_{0} \frac{\partial \boldsymbol{\Delta}_{a, \ell, t}}{\partial t}=k \frac{\partial^{2} \boldsymbol{\Delta}_{a, \ell, t}}{\partial \ell^{2}}-k h_{s} \boldsymbol{\Delta}_{a, \ell, t}+\mathbf{g}(a, \ell, t)
$$

The fluctuation $\boldsymbol{\Delta}_{\alpha, \ell, t}$ can be expanded as a series of eigenmodes (eq B1) in a similar manner to the case of unentangled stars, but with the difference in the eigenmode amplitudes because of the additional $-k h_{s} \boldsymbol{\Delta}_{a, \ell, t}$ term (compare the structure of eqs $4 \mathrm{a}$ and 9 ). In the remainder of this section, equations are presented in terms of tube coordinates by making the transformations [7] $s=\ell / N_{e}, a^{2}=N_{e} b^{2}$ (with $a$ the tube diameter) and $\widetilde{t}_{e}=\left|t-t^{\prime}\right| / \tau_{e}=\left(N_{a} / N_{e}\right)^{2} \widetilde{t}_{R_{a}}$.

Here, the procedure to obtain the MSD for the entangled stars is briefly described. For details regarding the derivations the reader is referred to Appendix B. First one works out the $\left\langle\boldsymbol{\Delta}_{\alpha, s, t} \cdot \boldsymbol{\Delta}_{\alpha, s^{\prime}, t^{\prime}}\right\rangle$ and $\left\langle\boldsymbol{\Delta}_{\alpha, s, t} \cdot \boldsymbol{\Delta}_{\beta, s^{\prime}, t^{\prime}}\right\rangle$ terms (eqs B3, B4 of Appendix B) by using equations B1 and B2. Since the chains in our theory are assumed to be Gaussian we know that at equilibrium $\left\langle\left(\mathbf{r}_{\alpha, s, 0}-\mathbf{r}_{\alpha, s^{\prime}, 0}\right)^{2}\right\rangle=a^{2}\left|s-s^{\prime}\right|$ and $\left\langle\left(\mathbf{r}_{\alpha, s, 0}-\mathbf{r}_{\beta, s^{\prime}, 0}\right)^{2}\right\rangle=$ $a^{2}\left(s+s^{\prime}\right)$. Therefore, the contribution of the mean path to the MSD can be calculated by using $\left\langle\left(\widehat{\mathbf{r}}_{\alpha, s}-\widehat{\mathbf{r}}_{\alpha, s^{\prime}}\right)^{2}\right\rangle=$ $a^{2}\left|s-s^{\prime}\right|-\left\langle\left(\boldsymbol{\Delta}_{\alpha, s, 0}-\boldsymbol{\Delta}_{\alpha, s^{\prime}, 0}\right)^{2}\right\rangle$ and $\left\langle\left(\widehat{\mathbf{r}}_{\alpha, s}-\widehat{\mathbf{r}}_{\beta, s^{\prime}}\right)^{2}\right\rangle=$ 
$a^{2}\left(s+s^{\prime}\right)-\left\langle\left(\boldsymbol{\Delta}_{\alpha, s, 0}-\boldsymbol{\Delta}_{\beta, s^{\prime}, 0}\right)^{2}\right\rangle$. For linear chains, these results agree with the mean path derived by Read [13]. Having evaluated the mean path contribution (eqs B6 of Appendix B) the final expressions are obtained using $\left\langle\left(\mathbf{r}_{\alpha, s, t}-\mathbf{r}_{\alpha, s^{\prime}, t^{\prime}}\right)^{2}\right\rangle=\left\langle\left(\widehat{\mathbf{r}}_{\alpha, s}-\widehat{\mathbf{r}}_{\alpha, s^{\prime}}\right)^{2}\right\rangle+\left\langle\left(\boldsymbol{\Delta}_{\alpha, s, t}-\boldsymbol{\Delta}_{\alpha, s^{\prime}, t^{\prime}}\right)^{2}\right\rangle$ and $\left\langle\left(\mathbf{r}_{\alpha, s, t}-\mathbf{r}_{\beta, s^{\prime}, t^{\prime}}\right)^{2}\right\rangle=\left\langle\left(\widehat{\mathbf{r}}_{\alpha, s}-\widehat{\mathbf{r}}_{\beta, s^{\prime}}\right)^{2}\right\rangle+\left\langle\left(\boldsymbol{\Delta}_{\alpha, s, t}-\right.\right.$
$\left.\left.\boldsymbol{\Delta}_{\beta, s^{\prime}, t^{\prime}}\right)^{2}\right\rangle$. The results are presented in Table II. The factor $k_{b}$ appearing in the expressions is equal to $N_{s} / N_{e}^{2}$. The appropriate selection for $k_{b}$, according to [13], is $k_{b}=1 / 4$.

TABLE II: MSD for entangled stars

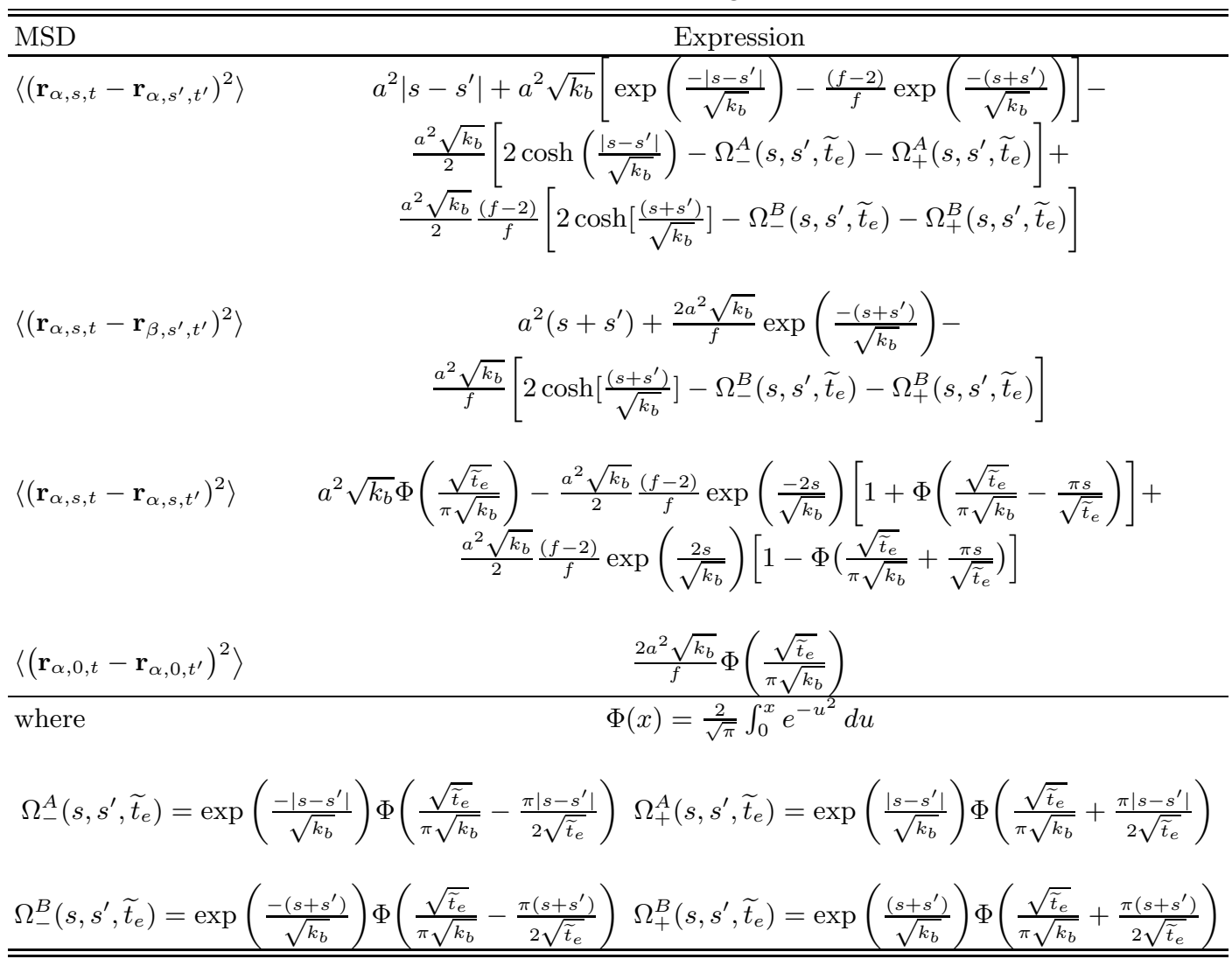

The top panel of Fig. 7 shows (dashed curves) our prediction for $\left\langle\left(\mathbf{r}_{\alpha, s, t}-\mathbf{r}_{\alpha, s, t^{\prime}}\right)^{2}\right\rangle / a^{2}$ as a function of $\widetilde{t}_{e}$, for three different segments along the arm. The blue, green and red dashed curves correspond to $s=0.05$ (near the branch point), $s=0.25$ and $s=1$ (one entanglement segment from the branch point), respectively. In the same panel, we plot (grey line) the MSD of the branch point of an unentangled star (fourth expression of table I), and the MSD of a segment of an unentangled linear chain (black line). The vertical shift between these two curves is $2 / f$. In order to obtain the grey and black lines we have converted the corresponding expressions for the MSD (Table I) in tube coordinates by using $\widetilde{t}_{R_{a}}=\widetilde{t}_{e}\left(N_{e} / N_{a}\right)^{2}, a^{2}=N_{e} b^{2}$ and $s=\ell N_{e}^{-1}$. In the bottom panel of Fig. 7 we plot the same MSD, normalized by $a^{2} \sqrt{\widetilde{t}_{e}}$, as in the top panel. We have also included the MSD of specific segments of unentangled stars, according to $\left\langle\left(\mathbf{r}_{\alpha, \ell, t}-\mathbf{r}_{\alpha, \ell, t^{\prime}}\right)^{2}\right\rangle$ of table I converted to tube coordinates. In analogy with the data sets for entangled stars, the blue, green, and red solid curves for the untentangled case correspond to $s=0.05, s=0.25$ and $s=1$, respectively.

The bottom panel of Fig. 7 shows that the segment closer to the branch point (blue dashed curve) follows at very early timescales, up to $\widetilde{t}_{e} \approx 0.01$, the segmental dynamics of an unentangled linear chain (black line). Then a crossover to the branch point dynamics of the unentangled case occurs, for timescales $0.02 \lesssim \widetilde{t}_{e} \lesssim 0.1$ (the dashed and solid blue lines coincide in that interval). At later timescales the segment starts to experience the localising effects and a final crossover to a plateau in the 

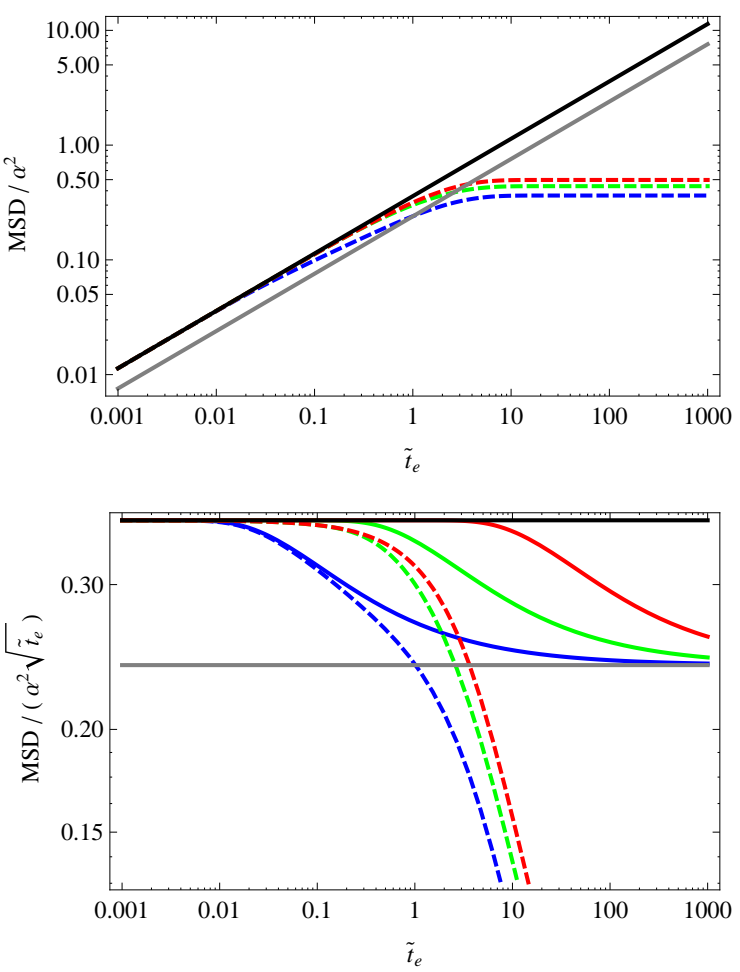

FIG. 7: Top: Segmental MSD in the entangled regime $\left(\left\langle\left(\mathbf{r}_{\alpha, s, t}-\mathbf{r}_{\alpha, s, t^{\prime}}\right)^{2}\right\rangle\right.$ of Table II), normalized by $a^{2}$, for three different segments along an arm: $s=0.05$ (blue curve), $s=0.25$ (green), and $s=1$ (red). The grey line corresponds to branch point motion in the unentangled regime while the black one to simple Rouse motion of an unentangled linear chain. Bottom: The same quantities as in the top panel, together with predictions for segmental motion in the unentangled regime $\left(\left\langle\left(\mathbf{r}_{\alpha, \ell, t}-\mathbf{r}_{\alpha, \ell, t^{\prime}}\right)^{2}\right\rangle\right.$ of Table I), represented as solid curves (colour codes correspond to same values of $s$ ). In this case the MSD has been normalized by $a^{2} \sqrt{\widetilde{t}_{e}}$. The expressions that refer to the unentangled case have been converted in tube coordinates using $\widetilde{t}_{R_{a}}=\widetilde{t}_{e} N_{e}^{2}\left(N_{a}^{2}\right)^{-1}, a^{2}=N_{e} b^{2}$ and $s=\ell\left(N_{e}\right)^{-1}$.

MSD occurs. This plateau arises at timescales $\widetilde{t}_{e} \gtrsim 1$ after the entanglement time, and shows up, as an horizontal line or a line of $-1 / 2$ slope, in the top and bottom panels of Fig. 7, respectively. The other two segments that are located further from the branch point $(s=0.25$ and $s=1$ ) are localised before they feel the presence of the branch point. Subsequently, their MSD exhibits a crossover from the unentangled linear chain behaviour to the plateau regime without following the branch point dynamics of the unentangled star.

At this point it must be reminded that we have assumed that motion is dominated by fast Rouse modes. Therefore, the expressions presented in Tables I and II are valid for timescales much smaller than the Rouse time of the arm, $\tau_{R_{a}}$, and for segments close to the central branch point. Thus, in the next Section we will limit the comparison between the theoretical MSD and the sim- ulation results to the case of the branch point, since it exhibits only a weak relaxation within the MD window (see Figs. 2 to 5).

\section{QUANTITATIVE EVALUATION OF MOLECULAR DYNAMICS DATA}

\section{A. Simulations with fixed chain ends}

As discussed in Section III (Figs. 2 to 5), in the MD simulations of entangled stars and Cayley trees several relaxation modes are active at different timescales. At early times $t<\tau_{e}$ the dynamics of the chain is dominated by Rouse motion. The Rouse regime are followed by local reptative motion ('Rouse in tube' dynamics) and by arm retraction depending on the position of the segment along the arm. Additionally, arm retraction contributes continuously to constraint release [15]. Since our theoretical expression for the segmental self-motion $\left(\left\langle\left(\mathbf{r}_{\alpha, s, t}-\mathbf{r}_{\alpha, s, t^{\prime}}\right)^{2}\right\rangle\right.$ of Table II) accounts only for internal Rouse modes its validity should be tested initially against MD simulations where all other relaxation mechanisms are to a high degree inactive. Accordingly, in the regime where such mechanisms are not effective we do not expect significant differences between the motion of the branch point in the simulated stars and that of the central branch point in the Cayley trees. In the remainder of the paper the data presented for the branch point motion in the Cayley tree must be understood as that of the central branch point.

With these ideas in mind, we have perfomed additional MD simulations of symmetric stars and Cayley trees in which the ends of the long arms $(Z=8)$ are fixed in space. This suppresses arm retraction, as well as constraint release driven by arm retraction, and therefore it provides information that can be directly compared with the theoretical results of Section IV B. The corresponding MSD, obtained from MD with fixed ends, for segments close to the branch point are shown in Fig. 8 with open symbols. For improving statistics, the MSD is averaged over ten monomers, namely the branch point and the three nearest monomers in each arm. For the remainder of the paper we shall refer to this group of ten monomers as 'the branch point' of the MD. As expected, the results confirm that on the time scales relevant for our combined study there are no differences, within statistics, between the MSD of the branch point in the star and Cayley tree (not shown).

Fig. 8 also includes our theoretical prediction as a red line. In a similar manner to the average perfomed in the MSD of the simulations, we have averaged over the MSD for the continuous chain between $s=0$ and $s=3 N_{e}^{-1}$ for each arm. The same procedure is performed in the comparison with the case of free ends (see Fig. 15 and explanation below). The theoretical MSD has been constructed by using the parameters $a^{2}=38$ and $\tau_{e}=1200$. We have forced such a value of $\tau_{e}$ in order to match the 


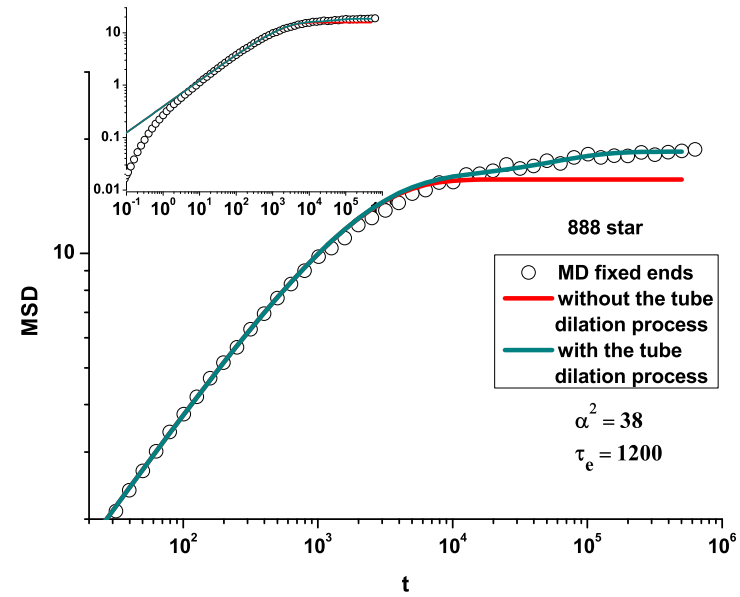

FIG. 8: MSD of the branch points of the stars with fixed ends. MD data are presented with black open symbols. The red line corresponds to the theoretical MSD without including the early tube dilation process. The thick cyan line shows the theoretical prediction when early tube dilation is taken into account.

theoretical and the simulation MSD in the Rouse regime (compare symbols and red line in Fig. 8 in the interval $\left.10 \lesssim t \lesssim 10^{3}\right)$. Obviously, since the model predicts Rouse dynamics at $t \rightarrow 0$, it does not account for the early ballistic motion observed in MD (see panel of Fig. 8). The theoretical entanglement time $\tau_{e}=1200$ is somewhat smaller than the value $\tau_{e}=1800$ estimated from the simulation MSD [3]. This difference might originate from local stiffness effects in the simulated chains (see Section II), which are not implemented in the model.

The most noticeable feature in Fig. 8 is that after the entanglement time of $\tau_{e} \sim 1200$, the MD data continue to rise whilst the theoretical MSD forms a clear plateau. As shown in Section IV B, the plateau in the theory is fully expected since no other relaxation mechanism, except internal Rouse motion, is included. In contrast, the MD data clearly indicate that, even if the arm ends are fixed, there is some relaxation of the entanglement constraints experienced by the branch point. This relaxation occurs after the branch point has explored its initial entanglement cage at the timescale $\tau_{e}$.

A possible interpretation of this observation is that there is some 'tube dilation' process occurring after $\tau_{e}$, giving rise to a slow relaxation of the tube localising potential. This tube dilation process occurs even in the absence of the 'standard' constraint release -i.e., the progressive dilution of the entanglement network mediated by the retraction of the arms. Indeed the mean path cannot relax since the arm ends are fixed. We can only speculate as to the mechanisms involved in this 'additional' constraint-release process. It could be due to tension equilibration along the constraining chains, which would occur at the Rouse time of the arms. In an earlier work Zhou and Larson [16] investigated, by MD of a similar bead-spring model, melts of linear chains with fixed chain ends. They also reported tube dilation and attributed it to a new type of constraint release, called 'end looping' constraint release (see Fig. 7 in Ref. [16]), which occurred through Rouse motion. However, for strongly entangled chains this process is only effective near the chain ends [16], and we do not expect it to be relevant for relaxation of the branch point in the systems investigated here.

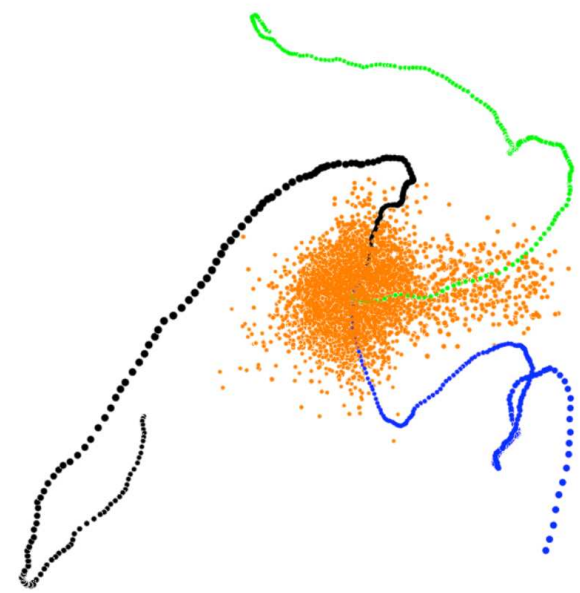

FIG. 9: For a selected star in the simulations with fixed ends, trajectory of the branch point (orange dots) and mean paths of the three arms (black, blue and green). Perspective depth is used. A deep fluctuation of the branch point along the green arm is clearly observed.

An alternative possibility is that the branch point makes short excursions along the tubes of each arm ('diving modes' [? ]), which in entropic terms are not so unfavourable as end looping. A precise characterization of the microscopic mechanisms involved in the early constraint release is beyond the scope of this work. Still, it is worth mentioning that visual inspection of branch point trajectories, in the MD simulations with fixed ends, gives some indications of the diving modes. Concretely, the branch point diving can be seen in roughly half of the branch point trajectories of Cayley tree and symmetric stars. Fig. 9 shows an example for a selected star. Orange dots represent the trajectory of the branch point (plotted at intervals of $\left.t \sim 0.1 \tau_{e}\right)$. The three curves formed by the black, blue and green lines are the 'mean paths' of the three arms. These have obtained by averaging the monomer positions over the whole trajectory of the simulation with fixed arms, and provide an estimation of the tube contour. The shape of the trajectory in the figure is not spherical and reveals a deep exploration of one of the tubes (green arm) by the branch point. Such a deep withdraw of the branch point in one particular direction occurs rarely, in the most cases the trajectory has an elliptical or triangular shape, indicating branch point excursions in two or three arm tubes.

Irrespective of its origin, we can quantify the magnitude of the effect of this process by treating it as a weak 
tube dilation, as follows. We assume that the tube enlargement depends weakly on time so there is a separation of timescale between fast Rouse motion within the tube and a slower "tube enlargement" process. Therefore, we may still use the expression for $\left\langle\left(\mathbf{r}_{\alpha, s, t}-\mathbf{r}_{\alpha, s, t^{\prime}}\right)^{2}\right\rangle$ in Table II after rescaling appropriately the model parameters as

$$
a^{2}(\Delta t)=\frac{a^{2}}{g(\Delta t)}, \tau_{e}(\Delta t)=\frac{\tau_{e}}{g^{2}(\Delta t)}, s(\Delta t)=s_{0} g(\Delta t)
$$

where $\Delta t=\left|t-t^{\prime}\right|$. Under this renormalisation the early Rouse-in-tube behaviour remains unchanged. The term $g(\Delta t)$ is a slowly varying tube dilation function which is obtained by minimising the error between the theory and the MD data using trial values in the range $[0,1]$. The so-obtained function $g(\Delta t)$ can be fitted to

$$
g(\Delta t)=g_{0}+g_{1} \exp \left(-\Delta t / \tau_{g}\right)
$$

with $g_{0} \approx 0.83, g_{1} \approx 1.5$, and $\tau_{g} \approx 41250$ for both stars and Cayley trees (since the respective MSD from simulations are identical within statistics). The cyan line in Fig. 8 represents the theoretical MSD of the branch point after incorporating the effect of tube dilation as described above. It is worth mentioning that $g(\Delta t)$ at the longest MD time approaches a value of 0.8 , which can be interpreted as an increase of the original tube diameter $a$ of the order of $10 \%$. The effective relaxation time for the tube dilation is comparable in magnitude to the Rouse time of the chains.

Having analyzed the mean square displacement for the branch point in the MD with fixed arm ends, we can move on to assess the effects of constraint release in the case of the free ends. We present our findings in the following subsection.

\section{B. Simulations with free chain ends}

Now we turn our attention to simulations with free ends (i.e. the 'standard' constraint release is now active). As aforementioned, it is well established that relaxation in symmetric star-like architectures does not occur by reptation, but via activated contour length fluctuations (CLF), also referred to as arm retraction [15]. When these fluctuations are deeper than an entanglement spacing the chain has to maneuver around the entanglements in order to fluctuate. This process is associated with an entropic penalty. As the CLF from the arm tip towards the branch point become deeper, the conformation that the chain has to adopt becomes entropically more unfavorable. This leads to a very broad distribution of relaxation times, as demonstrated in Figs. 2 to 5 . In particular, the separation of relaxation timescales along a star arm is exponential [15]. During arm retraction constraint release is also occuring. When chains escape from their original tubes (by arm retraction), they induce constraint release events on other chains. Thus, the molecular strands of the arms that are still unrelaxed (entangled) after a waiting time $t$, are able to explore a wider tube than the original one. Because of the broad spectrum of relaxation times, this tube dilation process due to $\mathrm{CR}$ is typically modeled as an effective dilution of the entanglement network (see section 4.3.2 of [15]). This approach is referred to as the 'dynamic dilution hypothesis' [15]. Our present simulations represent an opportunity to test this hypothesis.

For both studied systems the time $\tau_{a}$ taken for a complete retraction of the long arm $(Z=8)$ is not reached within the MD window. Thus, at the upper limit $\left(2 \cdot 10^{7} \tau_{o}\right)$ of the simulation time window there is an arm fraction of $\approx 0.3$ and $\approx 0.4$ in the star and the Cayley tree, respectively, that is still unrelaxed (see below). We take this fact into consideration and assume that branch point dynamics is still governed by chain motion within a localising potential at the end of the MD. If we make use of the dynamic dilution hypothesis, and take also into account the early tube dilation process discussed in subsection V A, we expect that the model parameters can be renormalized in this case as

$$
\begin{gathered}
a^{2}(\Delta t)=\frac{a^{2}}{g(\Delta t) \psi^{\alpha_{d}}(\Delta t)}, \\
\tau_{e}(\Delta t)=\frac{\tau_{e}}{g(\Delta t)^{2} \psi^{2 \alpha_{d}}(\Delta t)}, \\
s(\Delta t)=s_{0} g(\Delta t) \psi^{\alpha_{d}}(\Delta t),
\end{gathered}
$$

where $\psi(\Delta t)$ is the fraction of material that is still entangled (unrelaxed) after a waiting time $\Delta t$. The exponent $\alpha_{d}$ is the so-called dilution exponent (often assumed to be 1 or $4 / 3)$. In our calculations we have investigated both values of $\alpha_{d}$. The factor $g(\Delta t)$ is the function obtained in the previous section (eq 11) describing the early tube dilation process.

The dilution function $\psi(\Delta t)$ corresponds to the tube survival probability [15]. We can estimate it directly from the simulations as follows. Following the original work of Doi and Edwards [7], we formulate the tube survival probability in terms of the tangent correlation function and specify it for the case of a three-arm symmetric star:

$$
\psi_{\ell}(t)=\left\langle\mathbf{u}_{\alpha, \ell, 0} \cdot\left(\mathbf{R}_{\alpha, t}^{e}+B^{\prime} \mathbf{R}_{\beta, t}^{e}+C^{\prime} \mathbf{R}_{\gamma, t}^{e}\right)\right\rangle .
$$

In this equation $\mathbf{u}_{\alpha, \ell, 0}=\partial \mathbf{r}_{\alpha, \ell, 0} / \partial \ell$ represents the tangent vector at the $\ell$ th segment in the arm $\alpha$ at time 0 . $\mathbf{R}_{\alpha, t}^{e}=\mathbf{r}_{\alpha, N_{a}, t}-\mathbf{r}_{\alpha, 0, t}$ is the end-to-end vector of the arm $\alpha$ at time $t$. The three indices $\alpha, \beta, \gamma \in\{1,2,3\}$ are different and denote the three arms of the star. The numerical coefficients $B^{\prime}$ and $C^{\prime}$ provide the weight of the correlations between the arm $\alpha$ and the other two arms $\beta$ and $\gamma$.

Equations similar to eq 13 have been proposed [17], but using the mean path rather than the chain co-ordinates. The difference between these two approaches is that the mean path includes an average over short-time internal modes of the chain. We now demonstrate that, with suitably chosen coefficients $B^{\prime}$ and $C^{\prime}$, equation 13 does not 
decay due to local Rouse motion of the chain within the tube, and so it is not necessary to use the mean path. We demonstrate this by evaluating equation 13 for unentangled three-arm symmetric stars. First, we express the position vector $\mathbf{r}_{\alpha, \ell, t}$ in terms of the Rouse modes (see Appendix A, eq A2). Regarding the coefficients $B^{\prime}$, $C^{\prime}$, we consider first the case $B^{\prime}=C^{\prime}=-1 / 2$ ( 'halfcorrelation'). It can bee seen that, by inserting all these terms, eq 13 is transformed into

$$
\begin{gathered}
\psi_{\ell}(t)=\frac{4 b^{2}}{\pi} \sum_{p} \frac{1}{2 p-1} \times \\
{\left[\cos \left(\frac{(2 p-1) \pi s_{\ell}}{2}\right) \sin \left(\frac{(2 p-1) \pi}{2}\right) \exp \left(\frac{-\widetilde{t}_{R_{a}}(2 p-1)^{2}}{4}\right)\right],}
\end{gathered}
$$

where $s_{\ell}=\ell / N_{a}$ and $\widetilde{t}_{R_{a}}=t / \tau_{R_{a}}$. In Fig. 10 the equation 14 is plotted as a function of the normalized time $\widetilde{t}_{R_{a}}$ for different values of $s_{\ell}$. In the usual continuous approximation, the sum in the former equations extends from $p=1$ to $p=p_{\max } \rightarrow \infty$. For the numerical calculation we used $p_{\max }=1000$. Results for larger $p_{\max }$ were indistinguishable from those of Fig. 10. The figure shows that the function starts to decay only after all the segments from the arm end till $s_{\ell}$ segment have escaped from the tube, in fact around the Rouse time of (1- $\left.s_{\ell}\right)$ arm part. In particular, in case of the segment $s_{\ell}=0.15$ placed close to the branch point, the initial decay is seen a bit before the Rouse time of the arm. This indicates that the short lengthscale Rouse modes do not lead to decay of the function and therefore using the chain co-ordinates instead of the mean path has no significant effect on our calculations.

We also considered the option $B^{\prime}=C^{\prime}=-1$ ('fullcorrelation'). By including the full correlations between the star arms, the obtained function $\psi_{\ell}(t)$ exceeds the maximum expected value, i.e., $\psi_{\ell}(t)>1.0$ and gives undesirable peaks for segments close to the branch point $\left(s_{\ell}=0.15,0.3\right)$. To avoid this effect, in case of the symmetric star we take into account only the half-correlation $\left(B^{\prime}=C^{\prime}=-1 / 2\right)$ in eq 13 . The final choice of the prefactors for particular segments of the star and Cayley tree is illustrated in Fig. 11. Note that the arm of the Cayley tree is divided into three different parts (outer segments, inner segments, short arm), so there are more terms on the right side of the eq 13 for the Cayley tree and the indices $\alpha, \beta, \gamma$ denote in this case different parts of the molecule.

We move on to the entangled systems. We calculated the tangent correlation function (eq. 13) by using the simulation data. Thus, the tangent vector in eq. 13 was approximated by the end-to-end vector of an arm segment of length equal to ten monomeric units. This segment size was chosen as a compromise to both achieving good statistics and averaging fast monomer fluctuations (not captured within the coarse-grained tube model). In Fig. 12 we show the time evolution of the so-obtained correlation functions for different segments $s_{\ell}$ of the symmetric stars and Cayley trees. It is clear that not all the

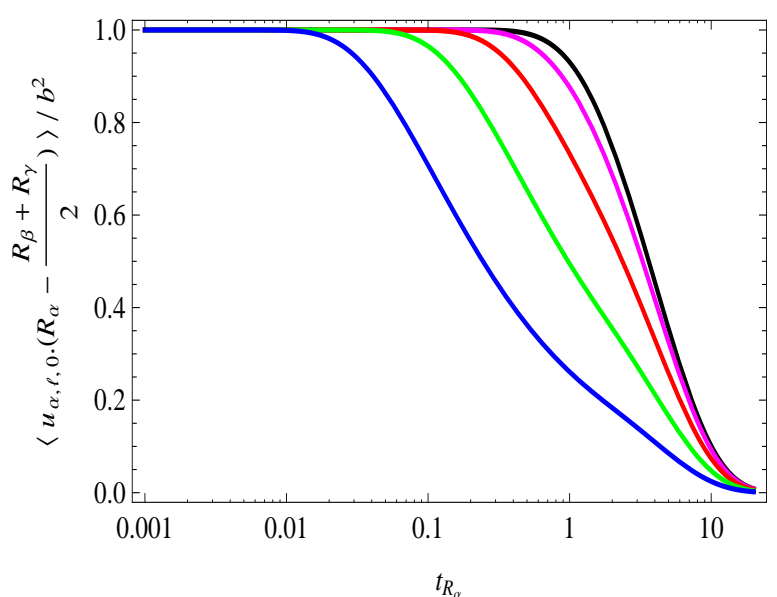

FIG. 10: Tangent correlation function (eq. 13) for unentangled stars. Half-correlation $\left(B^{\prime}=C^{\prime}=-1 / 2\right)$. Different colours correspond to different Rouse segments along the arm, $s_{l}=0.15$ (black), 0.3 (magenta), 0.5 (red), 0.7 (green), and 0.85 (blue).

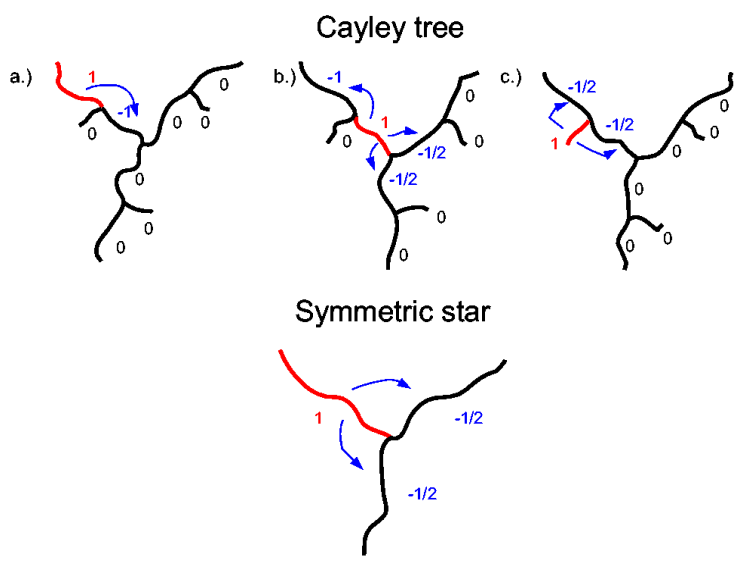

FIG. 11: Schematic representation of the correlations used for the correlator $\psi_{\ell}(t)$. Numbers labeling particular segments are the prefactors used in the equation 13 and red colour highlights the segment $\ell$. Figure a.) and b.) show the correlations of the outer and inner segments of the long arm of the Cayley tree, figure c.) illustrates the correlations of the short arm segments of the Cayley tree.

functions fully relax within the MD time window. Thus, the inner segments remain, on average, confined in their tubes - e.g., for $s_{\ell}=0.3$ the tube survival probability barely drops to the value $\psi_{\ell}(t)=0.8$ at the end of the simulation. The tangent correlators $\psi_{\ell}(t)$ were fitted to stretched exponential (Kohlraus-William-Watts, KWW) functions:

$$
\psi_{\ell}(t)=\exp \left(-\left(t / \tau_{\ell}\right)^{\beta}\right)
$$

where $\beta$ is the stretching exponent and $\tau_{\ell}$ is the relaxation time of the $\ell$ th-segment. From the fitting procedure 
we get a set of points $\left[s_{\ell} ; \tau_{\ell}\right]$, that provides us the information about the consecutive relaxation of the segments along the arms. We can use this information to construct functions $\Xi_{\alpha, \beta, \gamma}(t)$ that represent the fraction of unrelaxed material of the star arms or Cayley tree's parts $\alpha, \beta, \gamma$. The fuction is normalized so that $\Xi_{\alpha, \beta, \gamma}(0)=1$ (all the material is unrelaxed at $t=0$ ), and it decays with time in an exponential-like fashion, until all the material is relaxed, $\Xi_{\alpha, \beta, \gamma}\left(\tau_{N_{a}}\right)=1$.

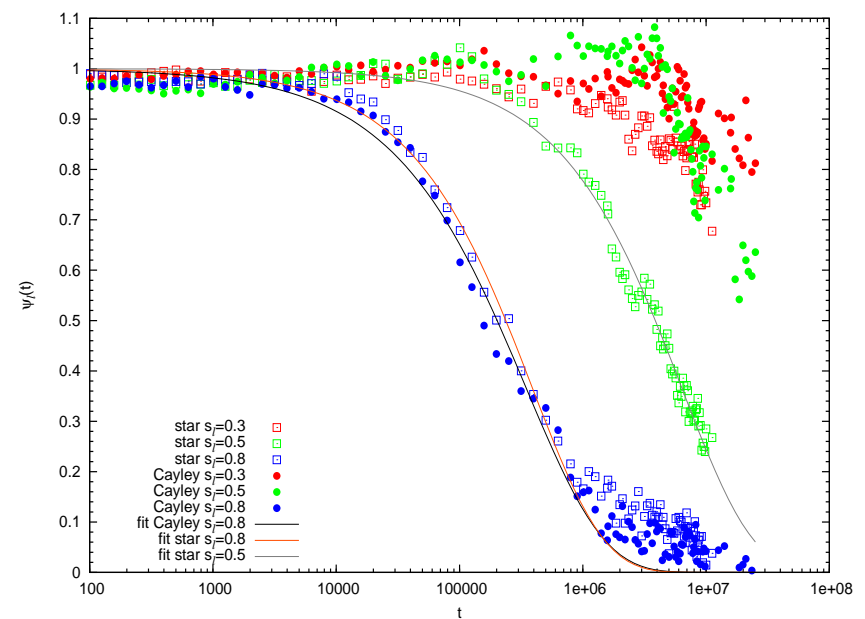

FIG. 12: Tangent correlation functions for the segments of symmetric star (squares) and Cayley tree (circles) with the fitting KWW functions

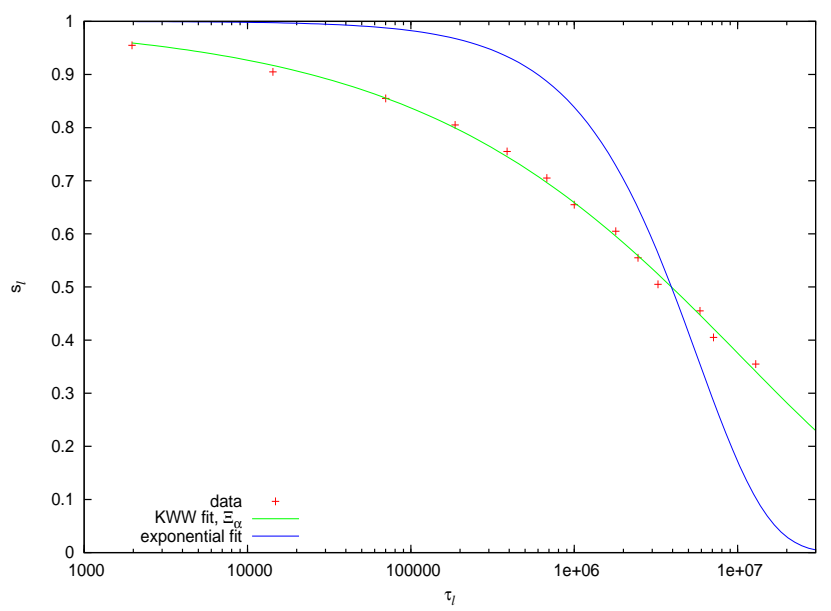

FIG. 13: Symbols: for the stars, KWW times of the tangent correlators $\psi_{\ell}(t)$ versus the respective coordinates $s_{\ell}$. Curves: fits to exponential (blue) and KWW behavior (green).

The procedure for obtaining the function $\Xi_{\alpha}(t)$ is illustrated in Fig. 13 for the case of the stars (obviously the function is identical for the three arms $\alpha, \beta, \gamma)$. There we show the coordinates $s_{\ell}$ versus the respective KWW times $\tau_{\ell}$, obtained from fitting the tangent correlators $\psi_{\ell}(t)$ as described above. This gives us a 'discrete' repre- sentation of the time elapsed for relaxing the fraction of the arm tube extending from $s=1$ (arm tip) to $s=s_{\ell}$. Likewise, the fraction from $s=s_{\ell}$ to $s=0$ (branch point) will be the fraction of unrelaxed tube at time $t=\tau_{\ell}$. By fitting the discrete set of points in Fig. 13 to some model function, we will obtain the continuous functions $\Xi_{\alpha, \beta, \gamma}(t)$ describing the tube survival probability of each $\operatorname{arm}\{\alpha, \beta, \gamma\}$. Two model functions have been used in the fit, namely an exponential (blue curve in Fig. 13) and a KWW function (green line). The data show strong deviations from pure exponential behaviour and are much better described by a KWW function. As aformentioned, in the star $\Xi_{\alpha}(t)$ is identical for the three arms. In the case of the Cayley tree, we have three sets of points $\left[s_{\ell} ; \tau_{\ell}\right]$ for each arm, since this consists of three parts: outer segments, inner segments, and short side branch. Thus, we perfomed fits for the three sets of data to obtain their (non-identical) functions $\Xi_{\alpha}(t)$.

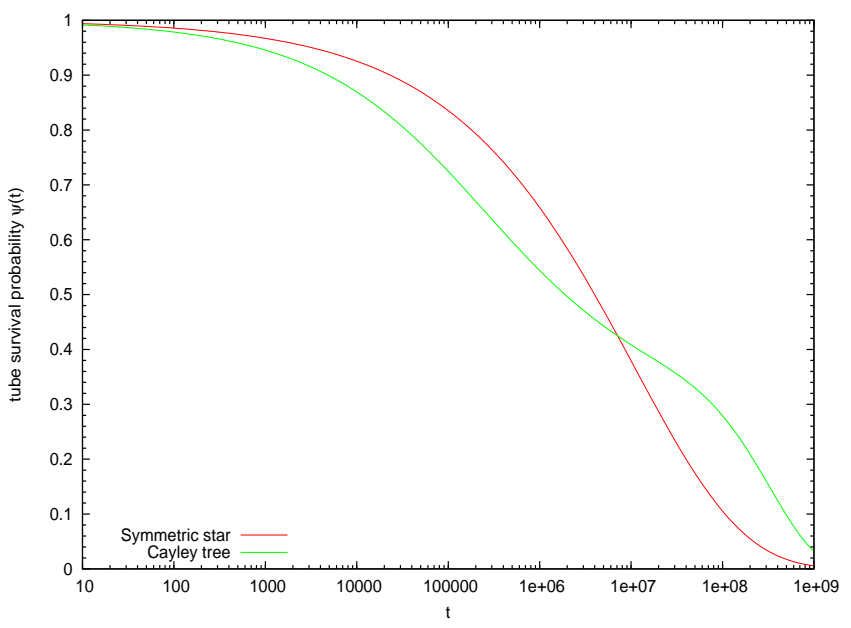

FIG. 14: Tube survival probability of the symmetric star and the Cayley tree, obtained from the simulations

Then, having the full description of the time evolution of the relaxation of the star arms and Cayley tree's parts, there is just a small step from the functions $\Xi_{\alpha}(t), \Xi_{\beta}(t), \Xi_{\gamma}(t)$ to the total tube survival probability $\Psi(t)$ of the star and Cayley tree. The total tube survival probability can be calculated as:

$$
\psi(t)=\frac{Z_{\alpha} \Xi_{\alpha}+Z_{\beta} \Xi_{\beta}+Z_{\gamma} \Xi_{\gamma}}{Z_{\alpha}+Z_{\beta}+Z_{\gamma}}
$$

where $Z_{\alpha}$ is the number of entanglements per star arm or Cayley tree's part $\alpha$. The functions $\psi(t)$ for the symmetric star and Cayley tree are shown in Fig. 14. Note that these model functions, constructed by following the fitting procedure described above, extend beyond the MD time window $\left(t \gtrsim 10^{7}\right)$, and fully decay at $t \sim 10^{9}$ but we note this is simply a possible extrapolation of the data. Nothing that follows depends on this. At the end of the $\mathrm{MD}$, the decay is $\psi(t) \approx 0.3$ and 0.4 for the star and Cayley tree, respectively. Interestingly, the fraction of unrelaxed material is larger in the star than in the Cayley tree 
up to time scales of $t \approx 7 \times 10^{6}$. Then the two curves $\psi(t)$ cross each other, and at longer times tube relaxation is much faster in the stars. The observed behavior seems consistent with an initially stronger tube dilution in the Cayley trees, being facilitated by the presence of the weakly entangled side branches. Indeed these relax in a time scale of about $t \approx 10^{6}$, i.e, roughly in the time window for which the stars show a higher $\psi(t)$. In hierarchical models, after their full relaxation the side branches act as friction points, and the Cayley trees reduce to an star containing 'fat beads' originating from the relaxed side branches. This additional friction slows down the retraction of the long arms, and relaxation of the Cayley tree at long times becomes slower than in the stars. This picture seems consistent with the trends observed in Fig. 14.

Finally, we use the tube survival probability estimated from the simulations to obtained the theoretical MSD of the branch point. The latter, as predicted by $\left\langle\left(\mathbf{r}_{\alpha, s, t}-\mathbf{r}_{\alpha, s, t^{\prime}}\right)^{2}\right\rangle$ of Table II, and using the rescaling parameters of eq 12, is represented in Fig. 15 with solid cyan lines. In the same figure the simulation data with free arm ends are depicted with open black circles. Data in top and bottom panels refer to the symmetric star and the Cayley tree, respectively. For comparison we include (small black crosses) the data of the simulation with fixed ends, previously presented in Fig. 8. Since constraint release is now active, the MSD of the branch point is larger than its counterpart in the MD with fixed ends (compare the open black circles and small black crosses for timescales bigger than $t \sim 10^{5} \tau_{0}$ ). From Fig. 15 it is clear that our theoretical prediction agrees very well with the MD data. Our results demonstrate that one can use the tube survival probability, parameterised by $\psi(t)$, to predict the effective dilution of the tube diameter, measured from the mean square displacement of the branch point. This observation is strong confirmation of the "dynamic dilution" hypothesis.

\section{CONCLUSIONS}

We have presented large-scale computer simulations of entangled polymers with symmetric star-like and Cayley tree-like architectures. Unlike the observation for reptation behaviour of linear chains, the simulated systems exhibit a strong dispersion, over several decades, of the relaxation times after the local reptative ('Rouse in tube') regime. In particular relaxation is dramatically slowed down when approaching the branch point from the outer segments. This is consistent with the expected retraction mechanism for relaxation of strongly entangled arms in branched aquitectures.

We have derived analytical expressions describing local motion of branched chains subject to entanglement constraints. The model provides a generalization of the Rouse regime for symmetric star-like architectures and introduces entanglements by localizing springs. Predic-
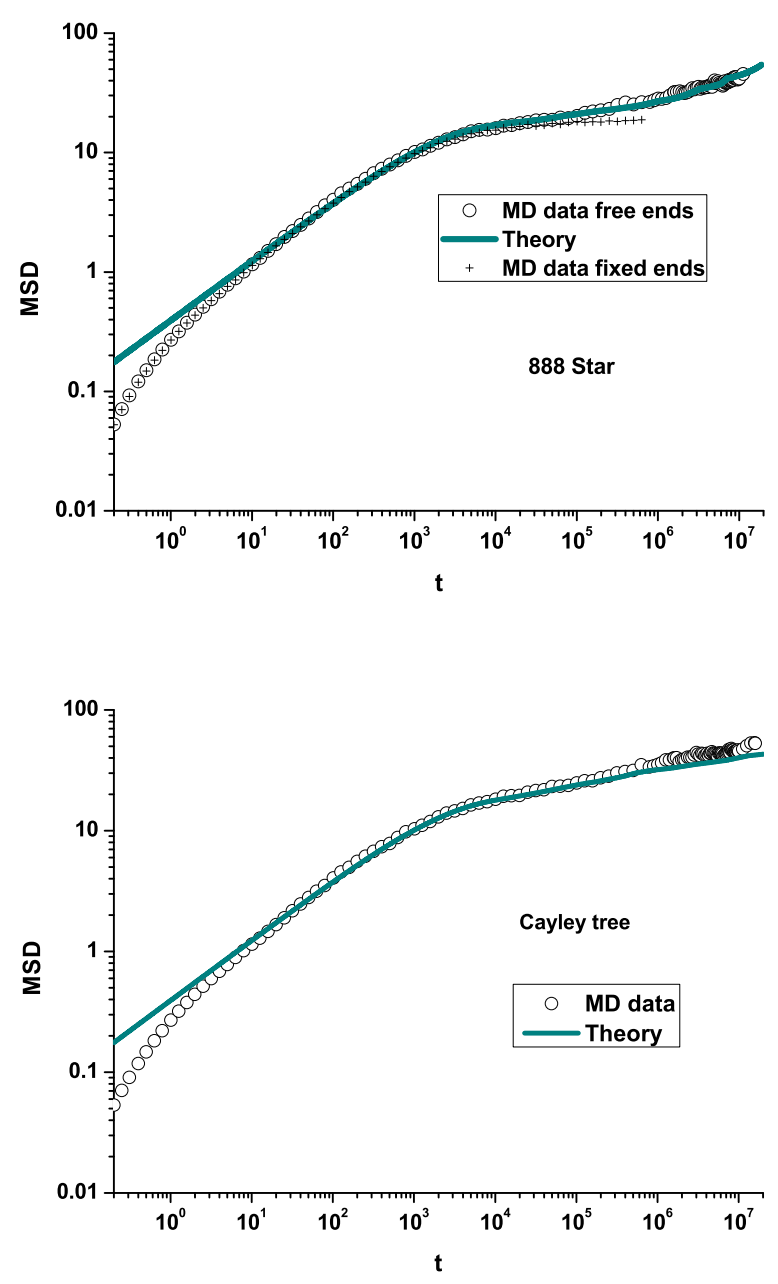

FIG. 15: MSD of the branch point of the symmetric stars (top) and Cayley trees (bottom) with free ends. MD data are represented with open black circles. The solid cyan curve corresponds to the theoretical MSD and accounts for CR events and the early tube dilation process. For comparison we also include the MD data for fixed arm ends (small black crosses).

tions of the model for localization of the branch point have been compared with simulations with fixed arm ends, which suppress retraction and constraint release. Strikingly, the simulations reveal that, in constrast with the model, the mean square displacement continues to grow weakly with increasing time, despite the absence of constraint-release effects. Possible mechanisms for this include tube dilution due to tension equilibration along surrounding chains, and branch point diving along the star-arm tubes (we have found some evidence of the latter). We have quantified the strength of this early tube dilation process, as well as the fraction of unrelaxed material in the simulation with free ends. This allows us to renormalize the tube diameter and entanglement time as time-dependent quantities. With this renormalization, our model provides an excellent description of the mean 
square displacement of the branch point. The fact that we were able to use the tube survival probability, measured from correlation functions within the simulation, to predict the effective dilution of the tube diameter, measured from the mean square displacement of the branchpoint, is strong confirmation of the physics underlying 'dynamic dilution' hypothesis.

Our analytical model can be used for making predictions for other observables. Thus, the first two expressions of Table II can be used for the calculation of the dynamic structure factor of a star, for example when treating experimental neutron spin echo data [18]. Work in this direction is in progress.

\section{ACKNOWLEDGEMENTS}

We specially acknowledge financial support from the project FP7-PEOPLE-2007-1-1-ITN (DYNACOP, Marie Curie Network, European Union), as well as from MAT2007-63681 (Spain) and IT-436-07 (GV, Spain). We acknowledge the programs PRACE, HPC-Europa2 and ESMI (European Union), and ICTS (Spain) for generous allocation of CPU time at GENCI (Paris, France), HLRS (Stuttgart, Germany), FZJ-JSC (Jülich, Germany), and CESGA (Santiago de Compostela, Spain).

\section{APPENDIX A: MSD UNENTANGLED REGIME}

The appropriate boundary conditions for a symmetric star with $f$ arms are

$$
\begin{aligned}
& \mathbf{r}_{\alpha=1, \ell=0, t}=\mathbf{r}_{\alpha=2, \ell=0, t}=\ldots=\mathbf{r}_{\alpha=f, \ell=0, t}+\ldots+\left.\frac{\partial \mathbf{r}}{\partial \ell}\right|_{\alpha=f, \ell=0}=0 \\
& \left.\frac{\partial \mathbf{r}}{\partial \ell}\right|_{\alpha=1, \ell=0}+\left.\frac{\partial \mathbf{r}}{\partial \ell}\right|_{\alpha=2, \ell=0}+\ldots(\mathrm{A} 1 \mathrm{a}) \\
& \left.\frac{\partial \mathbf{r}}{\partial \ell}\right|_{\alpha=1, \ell=N_{a}}=\left.\frac{\partial \mathbf{r}}{\partial \ell}\right|_{\alpha=2, \ell=N_{a}}=\ldots=\left.\frac{\partial \mathbf{r}}{\partial \ell}\right|_{\alpha=f, \ell=N_{a}}=0
\end{aligned}
$$

Equation A1a satisfies the chain connectivity requirement at the branch point while Eq. A1b represents the force balance at the branch point. Eq A1c indicates that there is no external force acting at the free ends of the arms. Under the previous boundary conditions, $\mathbf{r}_{\alpha, \ell, t}$ can be expressed as a series of eigenmodes, in particular one cosine eigenmode and $f^{\prime}=f-1$ sine eigenmodes, indexed with the mode numbers $p$ and $q$

$$
\begin{aligned}
\mathbf{r}_{\alpha, \ell, t}= & \sum_{p} \mathbf{X}_{p}^{c}(t) \Psi_{p}^{c}(\ell)+\sum_{q}\left(\mathbf{X}_{q}^{s_{1}}(t) \Psi_{q}^{s_{1}}(\alpha, \ell)+\right. \\
& \left.\ldots+\mathbf{X}_{q}^{s_{f^{\prime}}}(t) \Psi_{q}^{s_{f^{\prime}}}(\alpha, \ell)\right)
\end{aligned}
$$

with

$$
\begin{array}{r}
\Psi_{p}^{c}(\ell)=\cos \left(\frac{p \pi \ell}{N_{a}}\right) \\
\Psi_{q}^{s_{i}}(\alpha, \ell)=s_{i \alpha} \sin \left(\frac{(2 q-1) \pi \ell}{2 N_{a}}\right)
\end{array}
$$

The numerical coefficients $s_{i \alpha}$ satisfy the following constraints

$$
\begin{aligned}
& \sum_{\alpha=1}^{f} s_{i \alpha}=0 \\
& \sum_{\alpha=1}^{f} s_{i \alpha}^{2}=f \\
& \sum_{\alpha=1}^{f} s_{i \alpha} s_{j \alpha}=0
\end{aligned}
$$

where indices $i, j$ denote the $i t h$ and $j t h$ eigenmode respectively. Eq A4a is a consequence of the force balance at the branch point (eq A1b). Eqs A4b, A4c arise from normalization and orthogonality, respectively.

The first step towards the calculation of the MSD is to substitute eq A2 into eq $4 \mathrm{a}$ and operate from the left the resulting expression with the following integral operators

$$
\begin{gathered}
\sum_{\alpha=1}^{f} \int_{0}^{N_{a}} \sum_{p^{\prime}} \Psi_{p^{\prime}}^{c}(\ell) d \ell, \sum_{\alpha=1}^{f} \int_{0}^{N_{a}} \sum_{q^{\prime}} \Psi_{q^{\prime}}^{s_{1}}(\alpha, \ell) d \ell, \\
\ldots, \sum_{\alpha=1}^{f} \int_{0}^{N_{a}} \sum_{q^{\prime}} \Psi_{q^{\prime}}^{s_{f^{\prime}}}(\alpha, \ell) d \ell
\end{gathered}
$$


After making use of eqs A4 (only 'diagonal' terms, which contain products of the form $\Psi_{p^{\prime}}^{c} \Psi_{p}^{c}, \Psi_{q^{\prime}}^{s_{i}} \Psi_{q}^{s_{i}}$, are nonzero because of eqs $\mathrm{A} 4 \mathrm{a}$ and $\mathrm{A} 4 \mathrm{c}$ ), the following set of $f$ decoupled equations for the time evolution of the eigenmodes amplitudes $\mathbf{X}_{p}^{c}(t), \mathbf{X}_{q}^{s_{1}}(t), \ldots, \mathbf{X}_{q}^{s_{f^{\prime}}}(t)$ is obtained

$$
\begin{aligned}
& \zeta_{p} \frac{\partial \mathbf{X}_{p}^{c}(t)}{\partial t}=-k_{p}^{c} \mathbf{X}_{p}^{c}(t)+g_{p}^{c}(t) \\
& \zeta_{q} \frac{\partial \mathbf{X}_{q}^{s_{1}}(t)}{\partial t}=-k_{q}^{s_{1}} \mathbf{X}_{q}^{s_{1}}(t)+g_{q}^{s_{1}}(t) \\
& \zeta_{q} \frac{\partial \mathbf{X}_{q}^{s_{f^{\prime}}}(t)}{\partial t}=-k_{q}^{s_{f^{\prime}}} \mathbf{X}_{q}^{s_{f^{\prime}}}(t)+g_{q}^{s_{f^{\prime}}}(t)
\end{aligned}
$$

All equations that refer to sine eigenmodes are identical so in practice only two of the above equations need to be solved. In particular, the one that refers to $\mathbf{X}_{p}^{c}(t)$ and one of the remaining $f-1$ that refer to $\mathbf{X}_{q}^{s_{1}}(t)=\cdots=$ $\mathbf{X}_{q}^{s_{f^{\prime}}}(t)=\mathbf{X}_{q}^{s_{i}}(t)$. In the system of eqs A5, $\zeta_{p}=\zeta_{q}=$ $\frac{f \zeta_{0} N_{a}}{2}, k_{p}^{c}=\frac{f p^{2} \pi^{2} 3 k_{B} T}{2 N_{a} b^{2}}, k_{q}^{s_{i}}=\frac{f(2 q-1)^{2} \pi^{2} 3 k_{B} T}{8 N_{a} b^{2}}$ and

$$
\begin{aligned}
g_{p}^{c}(t) & =\sum_{\alpha=1}^{f} \int_{0}^{N_{a}} g(\alpha, \ell, t) \Psi_{p}^{c} d \ell \\
g_{q}^{s_{i}}(t) & =\sum_{\alpha=1}^{f} \int_{0}^{N_{a}} g(\alpha, \ell, t) \Psi_{q}^{s_{i}} d \ell
\end{aligned}
$$

The solutions for $\mathbf{X}_{p}^{c}(t)$ and $\mathbf{X}_{q}^{s_{i}}(t)$ are obtained using the integrating factor method and are

$$
\begin{aligned}
\mathbf{X}_{p}^{c}(t) & =\frac{\exp \left(-\frac{t}{\tau_{p}^{c}}\right)}{\zeta_{p}} \int_{-\infty}^{t} g_{p}^{c} \exp \left(\frac{t^{\prime}}{\tau_{p}^{c}}\right) d t^{\prime} \\
\mathbf{X}_{q}^{s_{i}}(t) & =\frac{\exp \left(-\frac{t}{\tau_{q}^{s_{i}}}\right)}{\zeta_{q}} \int_{-\infty}^{t} g_{q}^{s_{i}} \exp \left(\frac{t^{\prime}}{\tau_{q}^{s_{i}}}\right) d t^{\prime}
\end{aligned}
$$

where $\tau_{p}^{c}=\zeta_{p}\left(k_{p}^{c}\right)^{-1}$ and $\tau_{q}^{s_{i}}=\zeta_{q}\left(k_{q}^{s_{i}}\right)^{-1}$.

The next step is to compute the $\left\langle X_{p_{\mu}}^{c}(t) X_{p_{\nu}^{\prime}}^{c}\left(t^{\prime}\right)\right\rangle$ and $\left\langle X_{q_{\mu}}^{s_{i}}(t) X_{q_{\nu}^{\prime}}^{s_{i}}\left(t^{\prime}\right)\right\rangle$ averages. To do this, one first needs to work out the averages that involve the noise terms, namely $\left\langle g_{p_{\mu}}^{c} g_{p_{\nu}^{\prime}}^{c}\right\rangle$ and $\left\langle g_{q_{\mu}}^{s_{i}} g_{q_{\nu}^{\prime}}^{s_{i}}\right\rangle$ using eqs A6a-A6b and $\left\langle g_{\mu}(\alpha, \ell, t) g_{\nu}\left(\beta, \ell^{\prime}, t^{\prime}\right)\right\rangle=2 \zeta_{0} k_{B} T \delta\left(\ell-\ell^{\prime}\right) \delta\left(t-t^{\prime}\right) \delta_{\alpha \beta} \delta_{\mu \nu}$, and then use eqs A7a, A7b. The 'nondiagonal' averages of the form $\left\langle\mathbf{X}_{p_{\mu}}^{c}(t) \mathbf{X}_{q_{\nu}^{\prime}}^{s_{i}}\left(t^{\prime}\right)\right\rangle,\left\langle\mathbf{X}_{q_{\mu}}^{s_{i}}(t) \mathbf{X}_{q_{\nu}^{\prime}}^{s_{j}}\left(t^{\prime}\right)\right\rangle$ vanish since they contain orthogonal terms like $\Psi_{p}^{c} \Psi_{q^{\prime}}^{s_{i}}$ and $\Psi_{q}^{s_{i}} \Psi_{q^{\prime}}^{s_{j}}$. The final expressions are

$$
\begin{aligned}
& \left\langle\mathbf{X}_{p}^{c}(t) \cdot \mathbf{X}_{p^{\prime}}^{c}\left(t^{\prime}\right)\right\rangle=\frac{2 N_{a} b^{2} \delta_{p p^{\prime}}}{f \pi^{2} p^{2}} \exp \left(-\widetilde{t}_{R_{a}} p^{2}\right) \quad(\mathrm{A} 8 \mathrm{a}) \\
& \left\langle\mathbf{X}_{q}^{s_{i}}(t) \cdot \mathbf{X}_{q^{\prime}}^{s_{i}}\left(t^{\prime}\right)\right\rangle=\frac{8 N_{a} b^{2} \delta_{q q^{\prime}}}{f \pi^{2}(2 q-1)^{2}} \exp \left(\frac{-\widetilde{t}_{R_{a}}(2 q-1)^{2}}{4}\right)
\end{aligned}
$$

where $\widetilde{t}_{R_{a}}=\left|t-t^{\prime}\right| \tau_{R_{a}}^{-1}$. The timescale $\tau_{R_{a}}$ is the Rouse relaxation time of an arm of $N_{a}$ monomers and is given by $\tau_{R_{a}}=\zeta_{0} b^{2} N_{a}^{2}\left(3 \pi^{2} k_{B} T\right)^{-1}$. At equilibrium the previous expressions reduce to $\left\langle\mathbf{X}_{p}^{c}(0) \cdot \mathbf{X}_{p^{\prime}}^{c}(0)\right\rangle=\frac{2 N_{a} b^{2} \delta_{p p^{\prime}}}{f \pi^{2} p^{2}}$ and $\left\langle\mathbf{X}_{q}^{s_{i}}(0) \cdot \mathbf{X}_{q^{\prime}}^{s_{i}}(0)\right\rangle=\frac{8 N_{a} b^{2} \delta_{q q^{\prime}}}{f \pi^{2}(2 q-1)^{2}}$. We mention that $\left\langle\mathbf{X}_{p}^{c}(0) \cdot \mathbf{X}_{p^{\prime}}^{c}(0)\right\rangle$ and $\left\langle\mathbf{X}_{q}^{s_{i}}(0) \cdot \mathbf{X}_{q^{\prime}}^{s_{i}}(0)\right\rangle$ can be calculated by making use of the equipartition theorem, after substitution of eq A2 into the free energy of the system (eq 4b).

Having obtained the correlation functions of the mode amplitudes it is a straightforward procedure to obtain the expressions for the MSD. In order to obtain the final expressions for $\left\langle\left(\mathbf{r}_{\alpha, \ell, t}-\mathbf{r}_{\alpha, \ell^{\prime}, t^{\prime}}\right)^{2}\right\rangle$ and $\left\langle\left(\mathbf{r}_{\alpha, \ell, t}-\mathbf{r}_{\beta, \ell^{\prime}, t^{\prime}}\right)^{2}\right\rangle$ we need to calculate $\left\langle\mathbf{r}_{\alpha, \ell, t} \cdot \mathbf{r}_{\alpha, \ell^{\prime}, t^{\prime}}\right\rangle$ and $\left\langle\mathbf{r}_{\alpha, \ell, t} \cdot \mathbf{r}_{\beta, \ell^{\prime}, t^{\prime}}\right\rangle$. The results read

$$
\begin{aligned}
& \left\langle\mathbf{r}_{\alpha, \ell, t} \cdot \mathbf{r}_{\alpha, \ell^{\prime}, t^{\prime}}\right\rangle= \\
& \left\langle\left[\sum_{p} \mathbf{X}_{p}^{c}(t) \Psi_{p}^{c}(\ell)+\sum_{q}\left(\mathbf{X}_{q}^{s_{1}}(t) \Psi_{q}^{s_{1}}(\alpha, \ell)+\ldots+\right.\right.\right. \\
& \left.\left.\mathbf{X}_{q}^{s_{f^{\prime}}}(t) \Psi_{q}^{s_{f^{\prime}}}(\alpha, \ell)\right)\right] \cdot\left[\sum_{p^{\prime}} \mathbf{X}_{p^{\prime}}^{c}\left(t^{\prime}\right) \Psi_{p^{\prime}}^{c}\left(\ell^{\prime}\right)+\right. \\
& \left.\left.\sum_{q^{\prime}}\left(\mathbf{X}_{q^{\prime}}^{s_{1}}\left(t^{\prime}\right) \Psi_{q^{\prime}}^{s_{1}}\left(\alpha, \ell^{\prime}\right)+\ldots+\mathbf{X}_{q^{\prime}}^{s_{f^{\prime}}}\left(t^{\prime}\right) \Psi_{q^{\prime}}^{s_{f^{\prime}}}\left(\alpha, \ell^{\prime}\right)\right)\right]\right\rangle \\
= & \frac{N_{a} b^{2}}{\pi^{2}}\left[\int_{0}^{\infty} \frac{\cos \left(\frac{p \pi\left|\ell-\ell^{\prime}\right|}{N_{a}}\right)}{p^{2}} \exp \left(-\widetilde{t}_{R_{a}} p^{2}\right) d p-\right. \\
& \left.\left(\frac{f-2}{f}\right) \int_{0}^{\infty} \frac{\cos \left(\frac{p \pi\left(\ell+\ell^{\prime}\right)}{N_{a}}\right)}{p^{2}} \exp \left(-\widetilde{t}_{R_{a}} p^{2}\right) d p\right] \\
= & \frac{N_{a} b^{2}}{\pi^{1.5}} \sqrt{\widetilde{t}_{R_{a}}} \exp \left(-\frac{\pi^{2}\left|\ell-\ell^{\prime}\right|^{2}}{4 N_{a}^{2} \widetilde{t}_{R_{a}}}\right)- \\
& \frac{b^{2}\left|\ell-\ell^{\prime}\right|}{2} \Phi\left(\frac{\pi\left|\ell-\ell^{\prime}\right|}{\left.2 N_{a} \sqrt{\widetilde{t}_{R_{a}}}\right)+}\right. \\
& \frac{N_{a} b^{2}}{\pi^{1.5}}\left(\frac{f-2}{f}\right) \sqrt{\widetilde{t}_{R_{a}}} \exp \left(-\frac{\pi^{2}\left(\ell+\ell^{\prime}\right)^{2}}{4 N_{a}^{2} \widetilde{t}_{R_{a}}}\right)+ \\
& \frac{b^{2}\left(\ell+\ell^{\prime}\right)}{2}\left(\frac{f-2}{f}\right) \Phi\left(\frac{\pi\left(\ell+\ell^{\prime}\right)}{2 N_{a} \sqrt{\widetilde{t}_{R_{a}}}}\right)
\end{aligned}
$$


and

$$
\begin{aligned}
& \left\langle\mathbf{r}_{\alpha, \ell, t} \cdot \mathbf{r}_{\beta, \ell^{\prime}, t^{\prime}}\right\rangle= \\
& \left\langle\left[\sum_{p} \mathbf{X}_{p}^{c}(t) \Psi_{p}^{c}(\ell)+\sum_{q}\left(\mathbf{X}_{q}^{s_{1}}(t) \Psi_{q}^{s_{1}}(\alpha, \ell)+\ldots+\right.\right.\right. \\
& \left.\left.\mathbf{X}_{q}^{s_{f^{\prime}}}(t) \Psi_{q}^{s_{f^{\prime}}}(\alpha, \ell)\right)\right] \cdot\left[\sum_{p^{\prime}} \mathbf{X}_{p^{\prime}}^{c}\left(t^{\prime}\right) \Psi_{p^{\prime}}^{c}\left(\ell^{\prime}\right)+\right. \\
& \left.\left.\sum_{q^{\prime}}\left(\mathbf{X}_{q^{\prime}}^{s_{1}}\left(t^{\prime}\right) \Psi_{q^{\prime}}^{s_{1}}\left(\beta, \ell^{\prime}\right)+\ldots+\mathbf{X}_{q^{\prime}}^{s_{f^{\prime}}}\left(t^{\prime}\right) \Psi_{q^{\prime}}^{s_{f^{\prime}}}\left(\beta, \ell^{\prime}\right)\right)\right]\right\rangle \\
= & \frac{2 N_{a} b^{2}}{f \pi^{2}} \int_{0}^{\infty} \frac{\cos \left(\frac{p \pi\left(\ell+\ell^{\prime}\right)}{N_{a}}\right)}{p^{2}} \exp \left(-\widetilde{t}_{R_{a}} p^{2}\right) d p \\
= & -\frac{2 N_{a} b^{2}}{f \pi^{1.5}} \sqrt{\widetilde{t}_{R_{a}}} \exp \left(-\frac{\pi^{2}\left(\ell+\ell^{\prime}\right)^{2}}{4 N_{a}^{2} \widetilde{t}_{R_{a}}}\right)- \\
& \frac{b^{2}\left(\ell+\ell^{\prime}\right)}{f} \Phi\left(\frac{\pi\left(\ell+\ell^{\prime}\right)}{2 N_{a} \sqrt{\widetilde{t}_{R_{a}}}}\right)
\end{aligned}
$$

In the derivation of eqs A9, A10 we have used eqs A2, A8 and $s_{1 \alpha}^{2}+\ldots+s_{f^{\prime} \alpha}^{2}=f-1, s_{1 \alpha} s_{1 \beta}+\ldots+s_{f^{\prime} \alpha} s_{f^{\prime} \beta}=-1$. Moreover, we have approximated the sums as integrals and we have assumed $2 p-1=2 p, 2 q-1=2 q$, which physically means that the fast Rouse modes dominate the dynamics. For the evaluation of the integrals we have used

$$
\begin{aligned}
& \int_{0}^{\infty} \frac{\cos (\bar{A} x) \exp \left(-\bar{B} x^{2}\right)}{x^{2}} d x=-\sqrt{\pi \bar{B}} \exp \left(\frac{-\bar{A}^{2}}{4 \bar{B}}\right) \\
& -\frac{\bar{A} \pi}{2} \Phi\left(\frac{\bar{A}}{2 \sqrt{\bar{B}}}\right), \quad \bar{A}, \bar{B} \geq 0
\end{aligned}
$$

From eq A9, we arrive at

$$
\begin{aligned}
\left\langle\mathbf{r}_{\alpha, \ell, t} \cdot \mathbf{r}_{\alpha, l, t}\right\rangle & =\frac{(f-2)}{f} b^{2} \ell \\
\left\langle\mathbf{r}_{\alpha, \ell^{\prime}, t^{\prime}} \cdot \mathbf{r}_{\alpha, \ell^{\prime}, t^{\prime}}\right\rangle & =\frac{(f-2)}{f} b^{2} \ell^{\prime}=\left\langle\mathbf{r}_{\beta, \ell^{\prime}, t^{\prime}} \cdot \mathbf{r}_{\beta, \ell^{\prime}, t^{\prime}}\right\rangle
\end{aligned}
$$

By substituting eqs A9, A10, A11 into

$$
\begin{aligned}
\left\langle\left(\mathbf{r}_{\alpha, \ell, t}-\mathbf{r}_{\alpha, \ell^{\prime}, t^{\prime}}\right)^{2}\right\rangle & =\left\langle\mathbf{r}_{\alpha, \ell, t} \cdot \mathbf{r}_{\alpha, \ell, t}\right\rangle+\left\langle\mathbf{r}_{\alpha, \ell^{\prime}, t^{\prime}} \cdot \mathbf{r}_{\alpha, \ell^{\prime}, t^{\prime}}\right\rangle \\
& -2\left\langle\mathbf{r}_{\alpha, \ell, t} \cdot \mathbf{r}_{\alpha, \ell^{\prime}, t^{\prime}}\right\rangle \\
\left\langle\left(\mathbf{r}_{\alpha, \ell, t}-\mathbf{r}_{\beta, \ell^{\prime}, t^{\prime}}\right)^{2}\right\rangle & =\left\langle\mathbf{r}_{\alpha, \ell, t} \cdot \mathbf{r}_{\alpha, \ell, t}\right\rangle+\left\langle\mathbf{r}_{\beta, \ell^{\prime}, t^{\prime}} \cdot \mathbf{r}_{\beta, \ell^{\prime}, t^{\prime}}\right\rangle \\
& -2\left\langle\mathbf{r}_{\alpha, \ell, t} \cdot \mathbf{r}_{\beta, \ell^{\prime}, t^{\prime}}\right\rangle
\end{aligned}
$$

one obtains the first and second expressions of Table I.

\section{APPENDIX B: MSD ENTANGLED REGIME}

We start with the expansion of the fluctuation $\boldsymbol{\Delta}_{\alpha, \ell, t}$ as a series of eigenmodes, in particular cosine eigenmodes with degeneracy 1 and sine eigenmodes with degeneracy $f^{\prime}=f-1$, indexed with the mode numbers $p$ and $q$, respectively,

$$
\begin{aligned}
\boldsymbol{\Delta}_{\alpha, \ell, t}= & \sum_{p} \mathbf{Y}_{p}^{c}(t) \Psi_{p}^{c}(\alpha, \ell)+\sum_{q}\left(\mathbf{Y}_{q}^{s_{1}}(t) \Psi_{q}^{s_{1}}(\alpha, \ell)+\right. \\
& \left.\ldots+\mathbf{Y}_{q}^{s_{f^{\prime}}}(t) \Psi_{q}^{s_{f^{\prime}}}(\alpha, \ell)\right)
\end{aligned}
$$

The expansion is performed in a similar manner to eq A2 of Appendix A. The eigenmodes $\Psi_{p}^{c}$ and $\Psi_{q}^{s_{1}}, \ldots$, $\Psi_{q}^{s_{f^{\prime}}}$ are the same as in eq A2, and their explicit expressions are given by eqs A3. The eigenmode amplitudes $\mathbf{Y}_{p}^{c}$ and $\mathbf{Y}_{q}^{s_{i}}$ differ from those of the unentangled case $\left(\mathbf{X}_{p}^{c}\right.$ and $\left.\mathbf{X}_{q}^{s_{i}}\right)$ because of the additional $-k h_{s} \boldsymbol{\Delta}_{a, \ell, t}$ term in eq 9. The correlation functions $\left\langle\mathbf{Y}_{p}^{c}(t) \cdot \mathbf{Y}_{p^{\prime}}^{c}\left(t^{\prime}\right)\right\rangle$ and $\left\langle\mathbf{Y}_{q}^{s_{i}}(t) \cdot \mathbf{Y}_{q^{\prime}}^{s_{i}}\left(t^{\prime}\right)\right\rangle$, are calculated following exactly the same procedure as for the calculation of $\left\langle\mathbf{X}_{p}^{c}(t) \cdot \mathbf{X}_{p^{\prime}}^{c}\left(t^{\prime}\right)\right\rangle$ and $\left\langle\mathbf{X}_{q}^{s_{i}}(t) \cdot \mathbf{X}_{q^{\prime}}^{s_{i}}\left(t^{\prime}\right)\right\rangle$ in the unentangled case (see Appendix A). The final expressions are

$$
\begin{aligned}
& \left\langle\mathbf{Y}_{p}^{c}(t) \cdot \mathbf{Y}_{p^{\prime}}^{c}\left(t^{\prime}\right)\right\rangle=\frac{2 N_{a} b^{2} \delta_{p p^{\prime}}}{f \pi^{2}\left[p^{2}+\left(\sqrt{h_{s}} \frac{N_{a}}{\pi}\right)^{2}\right]} \times \\
& \exp \left(-\widetilde{t}_{R_{a}}\left[p^{2}+\left(\sqrt{h_{s}} \frac{N_{a}}{\pi}\right)^{2}\right]\right) \\
& \left\langle\mathbf{Y}_{q}^{s_{i}}(t) \cdot \mathbf{Y}_{q^{\prime}}^{s_{i}}\left(t^{\prime}\right)\right\rangle=\frac{8 N_{a} b^{2} \delta_{q q^{\prime}}}{f \pi^{2}\left[(2 q-1)^{2}+\left(2 \sqrt{h_{s}} \frac{N_{a}}{\pi}\right)^{2}\right]} \times \\
& \exp \left(-\frac{1}{4} \widetilde{t}_{R_{a}}\left[(2 q-1)^{2}+\left(2 \sqrt{h_{s}} \frac{N_{a}}{\pi}\right)^{2}\right]\right)
\end{aligned}
$$

In Appendix A we calculated the $\left\langle\mathbf{r}_{\alpha, \ell, t} \cdot \mathbf{r}_{\alpha, \ell^{\prime}, t^{\prime}}\right\rangle$ and $\left\langle\mathbf{r}_{\alpha, \ell, t} \cdot \mathbf{r}_{\beta, \ell^{\prime}, t^{\prime}}\right\rangle$ correlation functions. A similar calculation can be performed for $\left\langle\boldsymbol{\Delta}_{\alpha, \ell, t} \cdot \boldsymbol{\Delta}_{\alpha, \ell^{\prime}, t^{\prime}}\right\rangle$ and $\left\langle\boldsymbol{\Delta}_{\alpha, \ell, t} \cdot \boldsymbol{\Delta}_{\beta, \ell^{\prime}, t^{\prime}}\right\rangle$. The differences between the two cases are: one should use eqs B1, B2 instead of eqs A2, A8 and the final integrals are of different form. In the latter case one should use the following expression [19]

$$
\begin{aligned}
& \int_{0}^{\infty} \frac{\cos (\bar{A} x)}{x^{2}+\bar{C}^{2}} \exp \left(-\bar{B} x^{2}\right) d x=\frac{\pi}{4 \bar{C}} \exp \left(\bar{B} \bar{C}^{2}\right) \times \\
& {\left[2 \cosh (\bar{A} \bar{C})-\exp (-\bar{C} \bar{A}) \Phi\left(\bar{C} \sqrt{\bar{B}}-\frac{\bar{A}}{2 \sqrt{\bar{B}}}\right)-\right.} \\
& \left.\exp (\bar{C} \bar{A}) \Phi\left(\bar{C} \sqrt{\bar{B}}+\frac{\bar{A}}{2 \sqrt{\bar{B}}}\right)\right] \bar{A}, \bar{B} \geq 0, \bar{C}>0
\end{aligned}
$$

The results are expressed in tube coordinates using the transformation rules $\widetilde{t}_{R_{a}}=\widetilde{t}_{e} \frac{N_{e}^{2}}{N_{a}^{2}}, a^{2}=N_{e} b^{2}=$ 
$\sqrt{N_{s} k_{b}^{-1}} b^{2}\left(\right.$ recall that $\left.N_{s}=h_{s}^{-1}\right), s=\frac{\ell}{N_{e}}$ as

$$
\begin{aligned}
& \left\langle\boldsymbol{\Delta}_{\alpha, s, t} \cdot \boldsymbol{\Delta}_{\alpha, s^{\prime}, t^{\prime}}\right\rangle= \\
& \frac{a^{2} \sqrt{k_{b}}}{4}\left[2 \cosh \left(\frac{\left|s-s^{\prime}\right|}{\sqrt{k_{b}}}\right)-\frac{(f-2)}{f} 2 \cosh \left(\frac{\left(s+s^{\prime}\right)}{\sqrt{k_{b}}}\right)\right] \\
& -\frac{a^{2} \sqrt{k_{b}}}{4}\left[\Omega_{-}^{A}\left(s, s^{\prime}, \tilde{t}_{e}\right)+\Omega_{+}^{A}\left(s, s^{\prime}, \tilde{t}_{e}\right)\right]+ \\
& \frac{a^{2} \sqrt{k_{b}}}{4} \frac{(f-2)}{f}\left[\Omega_{-}^{B}\left(s, s^{\prime}, \tilde{t}_{e}\right)+\Omega_{+}^{B}\left(s, s^{\prime}, \tilde{t}_{e}\right)\right]
\end{aligned}
$$

and

$$
\begin{gathered}
\left\langle\boldsymbol{\Delta}_{\alpha, s, t} \cdot \boldsymbol{\Delta}_{\beta, s^{\prime}, t^{\prime}}\right\rangle=\frac{a^{2} \sqrt{k_{b}}}{2 f}\left[2 \cosh \left(\frac{\left(s+s^{\prime}\right)}{\sqrt{k_{b}}}\right)\right. \\
\left.-\Omega_{+}^{B}\left(s, s^{\prime}, \tilde{t}_{e}\right)-\Omega_{-}^{B}\left(s, s^{\prime}, \tilde{t}_{e}\right)\right]
\end{gathered}
$$

The functions $\Omega_{-}^{A}\left(s, s^{\prime}, \tilde{t}_{e}\right), \quad \Omega_{+}^{A}\left(s, s^{\prime}, \tilde{t}_{e}\right), \Omega_{-}^{B}\left(s, s^{\prime}, \tilde{t}_{e}\right)$, $\Omega_{+}^{B}\left(s, s^{\prime}, \tilde{t}_{e}\right)$ are defined in Table II. Moreover, from eq B3 we obtain

$$
\begin{aligned}
& \left\langle\boldsymbol{\Delta}_{\alpha, s, t} \cdot \boldsymbol{\Delta}_{\alpha, s, t}\right\rangle= \\
& \frac{a^{2} \sqrt{k_{b}}}{2}\left[1-\frac{(f-2)}{f} \exp \left(\frac{-2 s}{\sqrt{k_{b}}}\right)\right] \\
& \left\langle\boldsymbol{\Delta}_{\alpha, s^{\prime}, t^{\prime}} \cdot \boldsymbol{\Delta}_{\alpha, s^{\prime}, t^{\prime}}\right\rangle=\left\langle\boldsymbol{\Delta}_{\beta, s^{\prime}, t^{\prime}} \cdot \boldsymbol{\Delta}_{\beta, s^{\prime}, t^{\prime}}\right\rangle= \\
& \frac{a^{2} \sqrt{k_{b}}}{2}\left[1-\frac{(f-2)}{f} \exp \left(\frac{-2 s^{\prime}}{\sqrt{k_{b}}}\right)\right]
\end{aligned}
$$

From eqs B3, B4 and B5, $\left\langle\left(\boldsymbol{\Delta}_{\alpha, s, t}-\boldsymbol{\Delta}_{\alpha, s^{\prime}, t^{\prime}}\right)^{2}\right\rangle$ and $\left\langle\left(\boldsymbol{\Delta}_{\alpha, s, t}-\boldsymbol{\Delta}_{\beta, s^{\prime}, t^{\prime}}\right)^{2}\right\rangle$, and consequently their equilibrium expressions $\left(t=t^{\prime}=0\right)$, are easily obtained.
The chains in this model are considered Gaussian, thus at equilibrium $\left\langle\left(\mathbf{r}_{\alpha, s, 0}-\mathbf{r}_{\alpha, s^{\prime}, 0}\right)^{2}\right\rangle=a^{2}\left|s-s^{\prime}\right|$ and $\left\langle\left(\mathbf{r}_{\alpha, s, 0}-\mathbf{r}_{\beta, s^{\prime}, 0}\right)^{2}\right\rangle=a^{2}\left(s+s^{\prime}\right)$. Subsequently, the contribution of the mean path to the MSD can be calculated using

$$
\begin{aligned}
& \left\langle\left(\widehat{\mathbf{r}}_{\alpha, s}-\widehat{\mathbf{r}}_{\alpha, s^{\prime}}\right)^{2}\right\rangle=a^{2}\left|s-s^{\prime}\right|-\left\langle\left(\boldsymbol{\Delta}_{\alpha, s, 0}-\boldsymbol{\Delta}_{\alpha, s^{\prime}, 0}\right)^{2}\right\rangle \\
& \left\langle\left(\widehat{\mathbf{r}}_{\alpha, s}-\widehat{\mathbf{r}}_{\beta, s^{\prime}}\right)^{2}\right\rangle=a^{2}\left(s+s^{\prime}\right)-\left\langle\left(\boldsymbol{\Delta}_{\alpha, s, 0}-\boldsymbol{\Delta}_{\beta, s^{\prime}, 0}\right)^{2}\right\rangle
\end{aligned}
$$

The result is

for segments on the same arm:

$$
\begin{aligned}
& \left\langle\left(\widehat{\mathbf{r}}_{\alpha, s}-\widehat{\mathbf{r}}_{\alpha, s^{\prime}}\right)^{2}\right\rangle= \\
& a^{2}\left|s-s^{\prime}\right|-a^{2} \sqrt{k_{b}}\left[1-\exp \left(-\frac{\left|s-s^{\prime}\right|}{\sqrt{k_{b}}}\right)\right]+ \\
& \frac{a^{2} \sqrt{k_{b}}}{2}\left(\frac{f-2}{f}\right)\left[\exp \left(-\frac{s}{\sqrt{k_{b}}}\right)-\exp \left(-\frac{s^{\prime}}{\sqrt{k_{b}}}\right)\right]^{2}
\end{aligned}
$$

for segments on different arms:

$$
\begin{aligned}
& \left\langle\left(\widehat{\mathbf{r}}_{\alpha, s}-\widehat{\mathbf{r}}_{\beta, s^{\prime}}\right)^{2}\right\rangle= \\
& a^{2}\left(s+s^{\prime}\right)-a^{2} \sqrt{k_{b}}+\frac{2 a^{2} \sqrt{k_{b}}}{f} \exp \left(\frac{-\left(s+s^{\prime}\right)}{\sqrt{k_{b}}}\right)+ \\
& \frac{a^{2} \sqrt{k_{b}}}{2}\left(\frac{f-2}{f}\right)\left[\exp \left(-\frac{2 s}{\sqrt{k_{b}}}\right)+\exp \left(-\frac{2 s^{\prime}}{\sqrt{k_{b}}}\right)\right]
\end{aligned}
$$

To complete the derivation of the expressions for the MSD we use $\left\langle\left(\mathbf{r}_{\alpha, s, t}-\mathbf{r}_{\alpha, s^{\prime}, t^{\prime}}\right)^{2}\right\rangle=\left\langle\left(\widehat{\mathbf{r}}_{\alpha, s}-\widehat{\mathbf{r}}_{\alpha, s^{\prime}}\right)^{2}\right\rangle+$ $\left\langle\left(\boldsymbol{\Delta}_{\alpha, s, t}-\boldsymbol{\Delta}_{\alpha, s^{\prime}, t^{\prime}}\right)^{2}\right\rangle$ and $\left\langle\left(\mathbf{r}_{\alpha, s, t}-\mathbf{r}_{\beta, s^{\prime}, t^{\prime}}\right)^{2}\right\rangle=\left\langle\left(\widehat{\mathbf{r}}_{\alpha, s}-\right.\right.$ $\left.\left.\widehat{\mathbf{r}}_{\beta, s^{\prime}}\right)^{2}\right\rangle+\left\langle\left(\boldsymbol{\Delta}_{\alpha, s, t}-\boldsymbol{\Delta}_{\beta, s^{\prime}, t^{\prime}}\right)^{2}\right\rangle$. The results are presented in Table II.
[1] Kurt Kremer and Gary S. Grest. Dynamics of entangled linear polymer melts: A molecular-dynamics simulation. The Journal of Chemical Physics, 92(8):5057-5086, 1990.

[2] Ralf Everaers, Sathish K. Sukumaran, Gary S. Grest, Carsten Svaneborg, Arvind Sivasubramanian, and Kurt Kremer. Rheology and microscopic topology of entangled polymeric liquids. Science, 303(5659):823-826, 2004.

[3] Qiang Zhou and Ronald G. Larson. Direct molecular dynamics simulation of branch point motion in asymmetric star polymer melts. Macromolecules, 40(9):3443-3449, 2007.

[4] Zuowei Wang, Alexei E. Likhtman, and Ronald G. Larson. Segmental dynamics in entangled linear polymer melts. Macromolecules, 45(8):3557-3570, 2012.

[5] Rolf Auhl, Ralf Everaers, Gary S. Grest, Kurt Kremer, and Steven J. Plimpton. Equilibration of long chain polymer melts in computer simulations. The Journal of Chemical Physics, 119(24):12718-12728, 2003.

[6] Bernward A. Mann Hans-Jrg Limbach, Axel Arnold and Christian Holm. Espresso-an extensible simulation pack- age for research on soft matter systems. Comput. Phys. Commun, 174(9):704-727, AUG 142006.

[7] M. Doi and S. F. Edwards. The Theory of Polymer Dynamics. Oxford University Press, USA, November 1986.

[8] R. T. Deam and S. F. Edwards. The theory of rubber elasticity. Philos. Trans. Roy. Soc. London Ser. A, 280(1296):317-353, 1976.

[9] M. Warner and S.F. Edwards. Neutron scattering from strained polymer networks. Journal of Physics A: Mathematical and General, 11(8):1649-1655, 1978.

[10] T.A. Vilgis and F. Boue. Brownian motion of chains and crosslinks in a permanently linked network: The dynamic form factor. Journal of Polymer Science, Part B: Polymer Physics, 26(11):2291-2302, 1988.

[11] D.J. Read and T.C.B. McLeish. Microscopic theory for the "lozenge" contour plots in scattering from stretched polymer networks. Macromolecules, 30(20):6376-6384, 1997.

[12] B. Mergell and R. Everaers. Tube models for rubberelastic systems. Macromolecules, 34(16):5675-5686, 2001. 
[13] D.J. Read, K. Jagannathan, and A.E. Likhtman. Entangled polymers: Constraint release, mean paths, and tube bending energy. Macromolecules, 41(18):6843-6853, 2008.

[14] P.E. Rouse Jr. A theory of the linear viscoelastic properties of dilute solutions of coiling polymers. The Journal of Chemical Physics, 21(7):1272-1280, 1953.

[15] T.C.B. McLeish. Tube theory of entangled polymer dynamics. Advances in Physics, 51(6):1379-1527, 2002.

[16] Qiang Zhou and Ronald G. Larson. Direct calculation of the tube potential confining entangled polymers. Macromolecules, 39(19):6737-6743, 2006.

[17] Pavlos S. Stephanou, Chunggi Baig, Georgia Tsolou, Vlasis G. Mavrantzas, and Martin Kröger. Quantifying chain reptation in entangled polymer melts: Topological and dynamical mapping of atomistic simulation results onto the tube model. The Journal of Chemical Physics, 132(12):124904, 2010

[18] M. Zamponi, W. Pyckhout-Hintzen, A. Wischnewski, M. Monkenbusch, L. Willner, G. Kali, and D. Richten. Molecular observation of branch point motion in star polymer melts. Macromolecules, 43(1):518-524, 2010.

[19] I. S. Gradshteyn and I. M. Ryzhik. Table of integrals, series, and products. Elsevier/Academic Press, Amsterdam, seventh edition, 2007. Translated from the Russian, Translation edited and with a preface by Alan Jeffrey and Daniel Zwillinger. 